\title{
FER Performance Evaluation and Enhancement of IEEE 802.11 a/g/p WLAN over Multipath Fading Channels in GNU Radio and USRP N200 Environment
}

\author{
Muhammad Morshed Alam ${ }^{1}$, Mohammad Rakibul Islam ${ }^{1}$, Muhammad Yeasir Arafat ${ }^{2}$ and \\ Feroz Ahmed $^{2}$ \\ ${ }^{1}$ Department of Electrical and Electronic Engineering, Islamic University of Technology, Gazipur, Bangladesh \\ [e-mail: amorshed90@gmail.com, rakibultowhid@yahoo.com] \\ ${ }^{2}$ Department of Electrical and Electronic Engineering, Independent University, Bangladesh \\ [e-mail: yeasir.masud@gmail.com, fahmed@gmail.com] \\ *Corresponding author: Muhammad Morshed Alam
}

Received February 14, 2017; revised September 18, 2017; accepted October 12, 2017; published January 31, 2018

\begin{abstract}
In this paper, authors have been evaluated the Frame Error Rate (FER) performance of IEEE $802.11 \mathrm{a} / \mathrm{g} / \mathrm{p}$ standard $5 \mathrm{GHz}$ frequency band WLAN over Rayleigh and Rician distributed fading channels in presence of Additive White Gaussian Noise (AWGN). Orthogonal Frequency Division Multiplexing (OFDM) based transceiver is implemented by using realtime signal processing frameworks (IEEE 802.11 Blocks) in GNU Radio Companion (GRC) and Ettus USRP N200 is used to process the symbol over the wireless radio channel. The FER is calculated for each sub-carrier conventional modulation schemes used by OFDM such as BPSK, QPSK, 16, 64-QAM with different punctuated coding rates. More precise SNR is computed by modifying the SNR calculation process of YANS and NIST error rate model to estimate more accurate FER. Here, real-time signal constellations, OFDM signal spectrums etc. are also observed to find the effect of multipath propagation of signals through flat and frequency selective fading channels. To reduce the error rate due to the multipath fading effect and Doppler shifting, channel estimation (CE) and equalization techniques such as Least Square (LS) and training based adaptive Least Mean Square (LMS) algorithm are applied in the receiver. The simulation work is practically verified at GRC by turning into a pair of Software Define Radio (SDR) as a simultaneous transceiver.
\end{abstract}

Keywords: GNU Radio, USRP N200, OFDM, AWGN, Rayleigh and Rician Fading Channel, FER, LS, LMS 


\section{Introduction}

Software Define Radio (SDR) is a platform where the majority of the physical layer signal processing is done at software by using Field Programmable Gate Array (FPGA), General Purpose Processor (GPP) and Digital Signal Processor (DSP) instead of using complicated electrical hardware's [1]-[3]. GNU Radio is an open source widely used software define radio platforms [5] which has digital signal processing blocks such as signal sources, sinks, filters, modulators, demodulators, encoders, decoders, equalizers, synchronizers and much more [7], [26]. The critical signal processing blocks are developed in C++ language [1]; however basic flow graphs are generated in python language [5]. Using this software user can build their own applications to transmit or receive data with the help of Universal Software Radio Peripheral (USRP) devices associated Radio Frequency (RF) TX/RX ports [1], [7].

OFDM is widely used in almost all wireless communication standards such as cellular standards like LTE advance and WiMAX, digital broadcasting standards known as DVB-T, IEEE 802.11 WLAN standards etc [4], [10]. In OFDM, parallel high rate data symbols are loaded on comparatively low rate several numbers of closely spaced orthogonal sub-carriers [4]-[6] having safety guard interval between two adjacent symbols and each sub-carriers are performed with conventional digital modulation schemes such as BPSK, M-PSK, M-QAM [10] etc. For the precise symbol duration, the sub-carriers are independent to each other. As a result, for a particular center frequency of the symbol, only the corresponding sub-carriers have the peak power while others are zero [7], [19]. It provides higher resistance to multipath effects and gives high spectral efficiency [4]. IEEE 802.11 is basically set of a physical layer (PHY) and Media Access Control (MAC) layer specifications for wireless communication in 2.4, 5 and $60 \mathrm{GHz}$ frequency bands [5], [17]. There are several 802.11 WLAN standards are exists among them few are given at Table 1.

Table 1. IEEE 802.11 PHY Layer standards [23]

\begin{tabular}{|c|c|c|c|c|}
\hline $\begin{array}{c}\text { Wireless LAN } \\
\text { standard }\end{array}$ & $\begin{array}{l}\text { Radio Frequency } \\
\text { Band (GHz) }\end{array}$ & Modulation & $\begin{array}{l}\text { Bandwidth } \\
\text { (MHz) }\end{array}$ & $\begin{array}{c}\text { Highest } \\
\text { Conceivable } \\
\text { Data Rate } \\
\text { (Mbps) }\end{array}$ \\
\hline IEEE 802.11 a & 5 & OFDM & 20 & 54 \\
\hline IEEE 802.11 g & 2.4 & OFDM & 20 & 54 \\
\hline IEEE 802.11 p & 5 & OFDM & 10 & 54 \\
\hline IEEE 802.11 ac & 5 & MIMO-OFDM & $20,40,80,160$ & $700-800$ \\
\hline
\end{tabular}

In wireless communication, multipath fading effect causes time and frequency dispersion of wireless radio signals as the mobile devices are not stationary [6]. These effects have the impact on the FER of any modulation schemes as mobile radio channels cause the significant fade or distort of the transmitted signal with compared to AWGN channel [9], [11]. Channel Estimation (CE) and equalization techniques can be applied to reduce the FER [8] and Inter Symbol Interference (ISI) created by multipath fading effects [13]-[14]. For a particular channel, ISI is introduced as the modulation bandwidth exceeds the coherence bandwidth due to the pulse broadening in time domain [11], [17]. As the mobile fading channel characteristics are random and time varying nature [11], the equalizers must have the adaptive characteristics [9] to track with the time varying components [14], [17]. At the 
beginning, a fixed length predefined training symbols are periodically sent by the transmitter that is known to the receiver so that the estimator can adapt to a minimum FER detection function and have the idea of the channel impulse response behavior caused by multipath [15]. Then the user's data symbols are transmitted following by the periodic training symbols [17] since the equalizer has the adaptive characteristics [16] it utilizes the adaptive Least Mean Square (LMS) technique to estimate the desired filter coefficients in order to compensate the distortion created by the multipath [3]. These predefined tanning symbols help the receiver end estimator to update with the suitable filter coefficients on worse case channel condition or even on rapidly time varying channel condition [19]-[20]. When the training sequences are finished the filter coefficients are updated with nearly optimum values through a continuous consequential adaptive algorithm process to get the convergence of the estimator.

\section{OFDM Overview}

Due to high spectral efficiency and robust performance at multipath fading channel OFDM is widely used in wireless communication. Let us consider, $x_{1}, x_{2}, \ldots \ldots, x_{N}$ are the data symbols in the frequency domain. These data symbols are transmitted to the IDFT operation after inserting the OFDM pilot symbols. Therefore, for the $k^{\text {th }}$ data symbol the corresponding time domain data signal $x(n)$ is given below [1], [4]:

$$
x(n)=\sum_{k=0}^{N-1} x(k) e^{\frac{j 2 \pi k n}{N}}, \quad 0 \leq n \leq N-1
$$

Here, $\mathrm{N}$ is the total number of sub-carriers. $\sum_{n=1}^{N} x(n)^{k}\left(x(n)^{j}\right)^{*}=0$, here $\mathrm{k} \neq \mathrm{j}$ and $*$ denotes the complex conjugate of the $j^{t h}$ sample. This equation confirms the orthogonal property of N OFDM sub-carriers to avoid Inter Carrier Interference (ICI) between them. The guard interval is provided between each time domain data symbols to avoid the ISI in OFDM system. Let the time domain samples $x(n)$ turn into $x_{G}(n)$ after the insertion of the guard interval and that should be transmitted over the wireless channel. The resulting transmitted symbol is:

$$
x_{G}(n)=\left\{\begin{array}{lr}
x(N+n), & n=-N_{G},-N_{G}+1, \ldots \ldots,-1 \\
x(n), & n=0,1, \ldots, N-1
\end{array}\right.
$$

Here, $N_{G}$ is the number samples in the guard interval. The transmitted signal will propagate through the frequency selective multipath fading wireless channel. Therefore transmitted symbols can be modulated as a circular convolution between channel impulse response and transmitted data symbols. Let the receiver got the received signal as $y_{G}(n)$ [4] expressed as given below:

$$
y_{G}(n)=x_{G}(n) * h(n, \tau)+w(n)=\int h(n, \tau) x_{G}(n-\tau) d \tau+w(n)
$$

Here, $h(n, \tau)$ is the impulse response of the fading channel, $w(n)$ is the additive white Gaussian noise. Channel impulse response $h(n, \tau)$ consisting of $L$ paths can be expressed as given below [6]:

$$
h(n, \tau)=\sum_{j=0}^{L-1} h_{j} e^{j 2 \pi f_{D j} t_{\bar{N}}^{n}} \delta\left(n-\tau_{j}\right), 0 \leq n \leq N-1=\sum_{j=0}^{L-1} a_{n} \delta\left(n-\tau_{j}\right)
$$


Here, $L$ is the total number of possible propagation paths, $f_{D j}$ is the $j^{\text {th }}$ path Doppler frequency shift which creates ICI of the received signals, $h_{j}$ is the complex impulse response of the $j^{t h}$ path and $\tau_{j}$ is the $j^{\text {th }}$ path time delay and $a_{n}$ is the complex path attenuation factor which gives the time varying complex amplitude, defined as the exponential term $a_{n}=$ $h_{j} e^{j 2 \pi f_{D j} t \frac{n}{N}}$. Channel Doppler shift is chosen as per the jakes spectrum or a flat spectrum, $\left[-f_{D \max }, f_{D \max }\right]$, here maximum Doppler frequency $f_{D}=\frac{v f_{c}}{c}$. Normalized Doppler frequency is defined as $f_{D} T_{S}$, where $T_{S}$ is the symbol period. We assume that the delay spreads are shorter than the cyclic prefix which allows reducing ISI between OFDM symbols.

After excluding the safety guard interval from $y_{G}(n)$, the received signal turns into $y(n)$ which are sent for the DFT operation to de multiplex the multi carrier signals and to get back again frequency domain data samples which have been transmitted over the fading channel [6], [10].

$$
Y(k)=\left[\frac{1}{N} \sum_{n=0}^{N-1} y(n) e^{\frac{-j 2 \pi k n}{N}}\right], 0 \leq k \leq N-1
$$

Finally, at the receiver end output the de multiplexed signal can be expressed as given below:

$$
Y(k)=X(k) H(k)+W(k), \quad 0 \leq k \leq N-1
$$

Here $X(k)$ is the transmitted signal, $H(k)$ is the channel transfer function represented in the frequency domain and $W(k)$ is the FFT of Gaussian noise $w(n)$.

\section{IEEE 802.11 a/g/p Transceiver Model at GNU Radio Companion}

IEEE $802.11 \mathrm{a} / \mathrm{g} / \mathrm{p}$ standard transmitter specifies total 64 sub-carriers $(\mathrm{N})$ for the $20 \mathrm{MHz}$ (802.11 a/g) or $10 \mathrm{MHz}$ bandwidth (802.11p), among them data sub-carriers $N_{D}=48$ are used for data, 4 pilot sub-carriers $N_{P}$ are assigned between data sub-carriers as per comb pilot insertion method to assist receiver in frequency offset detection and compensation, rest 12 sub-carriers are kept as null $N_{0}$ which is used to avoid Direct Current (DC) offset and frequency leakage to other frequency bands [1]-[3]. In case of every non-null sub-carrier first two symbols are kept for pilots were known as block type pilot symbols allocation to estimate channel coefficient. This type of carrier allocation known as block-comb combination method and it is very efficient to deal with quasi-static channels [10].

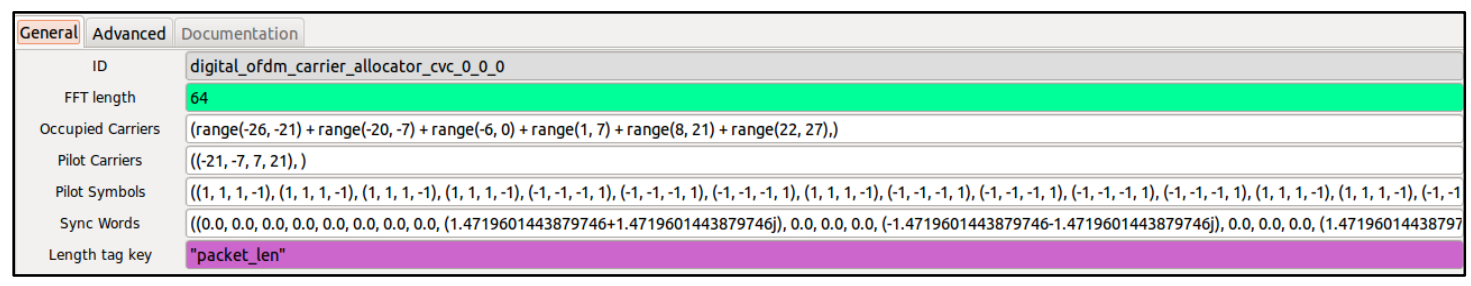

Fig. 1. IEEE 802.11 a/g/p OFDM sub-carrier allocator configuration [22] 


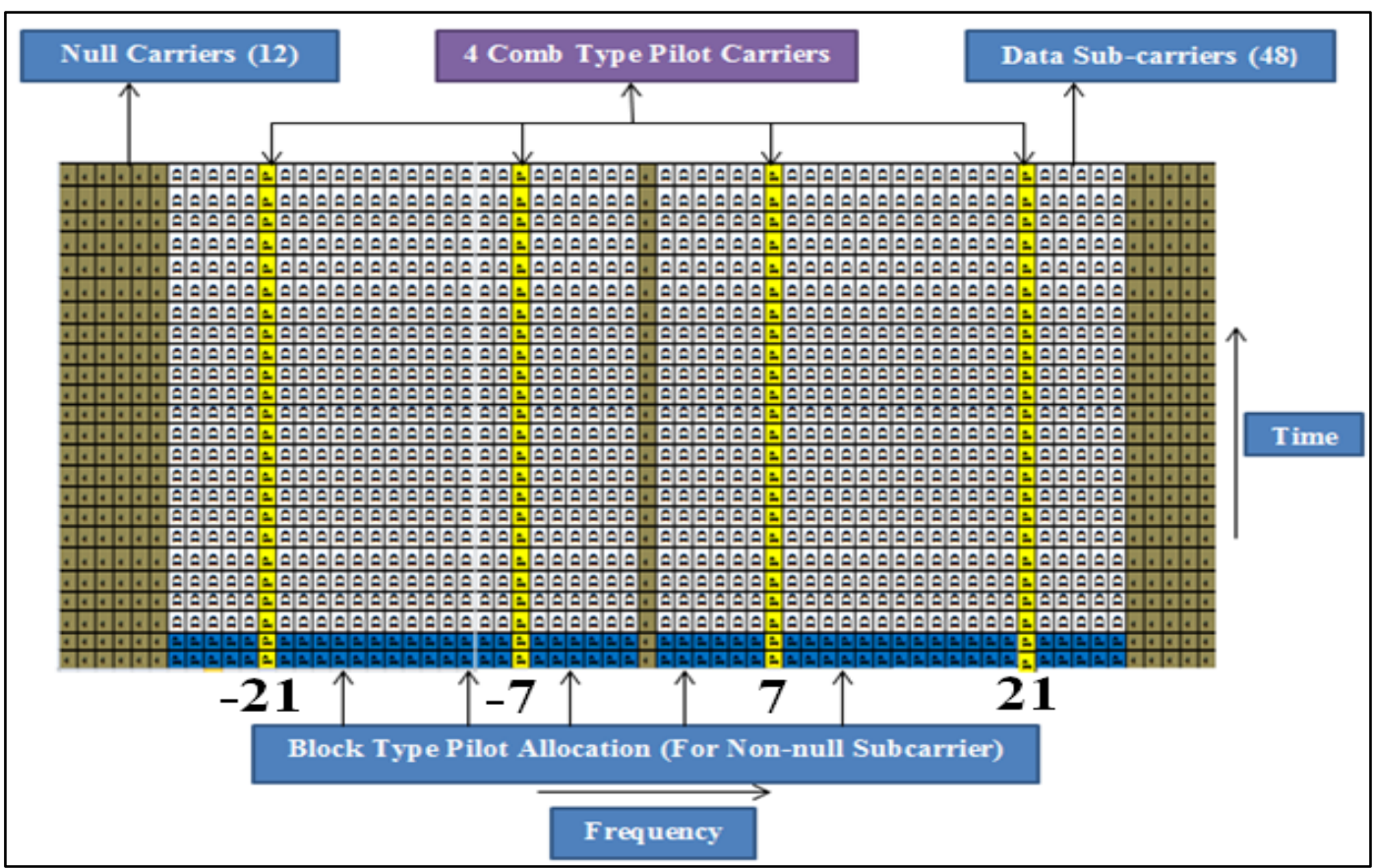

Fig. 2. Sub-carrier's allocation and pilot's insertion (comb and block types) in IEEE 802.11p

The receiver will get multiple copies of the signal with different strength even at the different time due to the reflection, scattering of the transmitted signal over the fading channels which may introduce the frequency offset in the sub-carriers. As a result, they may lose the orthogonal property resulting inter carrier interference. To remove the multipath effects guard interval $N_{G}$ known as a cyclic prefix is used at the beginning of each OFDM symbol. The last $N_{G}=16$ samples are copied from the time domain OFDM symbol $x$ and put at the beginning of the $x$. As a result, $N+N_{G}=80$ samples $\left(x_{N-N_{G}+1}, \ldots \ldots, x_{N}, x_{1}, \ldots \ldots, x_{N}\right)$ are transmitted per OFDM symbol [12].

The physical layer is encapsulated in WIFI PHY Hierarchical block which is the integrated systems for the transmitter and receiver in GNU Radio Companion given at Fig. 3.
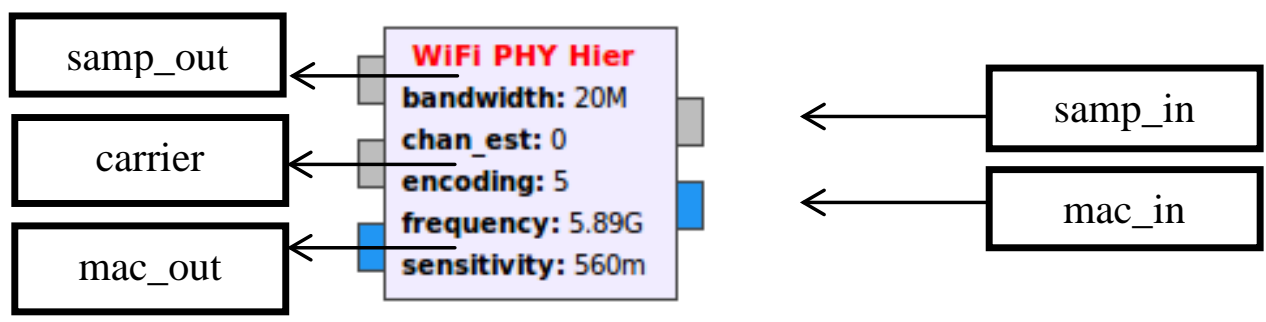

Fig. 3. IEEE 802.11 WIFI PHY HIER Block (TX and RX)

Basically, the transmitter consists of an encoder, OFDM carrier allocator, Inverse Fast Fourier Transform (IFFT) operation and cyclic prefix adder. In hierarchical block, WIFI Mapper is responsible for data scrambling, interleaving and convolution encoding. Packet Header Generator generates the header of the frame including the signal and services field. 


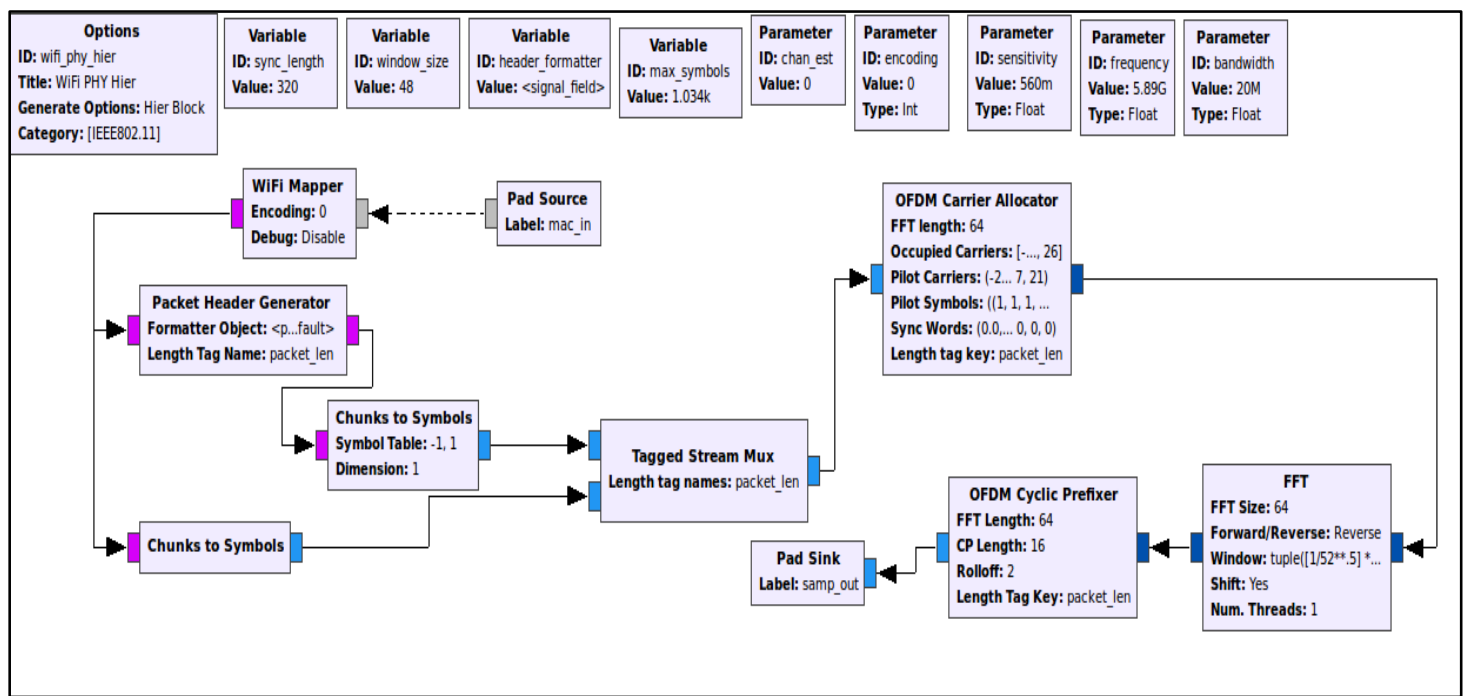

Fig. 4. IEEE 802.11 standard packet transmitter [22]

The frame header is BPSK modulated by the upper chunks to symbols block (symbol table: $1,-1$ ) and the remaining frame is modulated by the bottom chunks to symbols block as per the preferred modulation schemes. Finally, the header is integrated to the remaining of the frame by using Tagged stream MUX block. Then, multiplexed frames are transmitted to the OFDM carrier allocator block for the insertion of pilot sub-carriers uniformly inside the data sub-carriers and IFFT block is used to convert the symbols from frequency domain to the time domain. At last, OFDM Cyclic Prefixer block is used to add guard interval in the time domain before each OFDM symbol of the frames to avoid ISI. A short OFDM training symbols consist of 12 sub-carriers which are given below [23]:

$$
\begin{aligned}
& S_{-26,26} \\
& =\sqrt{\frac{13}{6}}\left[\begin{array}{c}
0,0,1+j, 0,0,0,-1-j, 0,0,0,1+j, 0,0,0,-1-j, 0,0,0,-1-j, 0,0,0,1+j, 0,0,0,0,0,0,0,-1-j, 0 \\
0,0,-1-j, 0,0,0,1+j, 0,0,0,1+j, 0,0,0,1+j, 0,0,0,1+j, 0,0
\end{array}\right]
\end{aligned}
$$

The factor $\sqrt{\frac{13}{6}}$ is used to normalize the average power of the OFDM symbol. The predefined long training symbols are 53 sub-carriers and it has a zero value at DC. The long training symbols are given below [23]:

$$
\begin{aligned}
& L_{-26,26} \\
& =[1,1,-1,-1,1,1,-1,1,-1,1,1,1,1,1,1,-1,-1,1,1,-1,1,-1,1,1,1,1,0,1,-1,-1,1,1,-1,1,-1,1,-1, \\
& \quad-1,-1,-1,-1,1,1,-1,-1,1,-1,1,-1,1,1,1,1]
\end{aligned}
$$




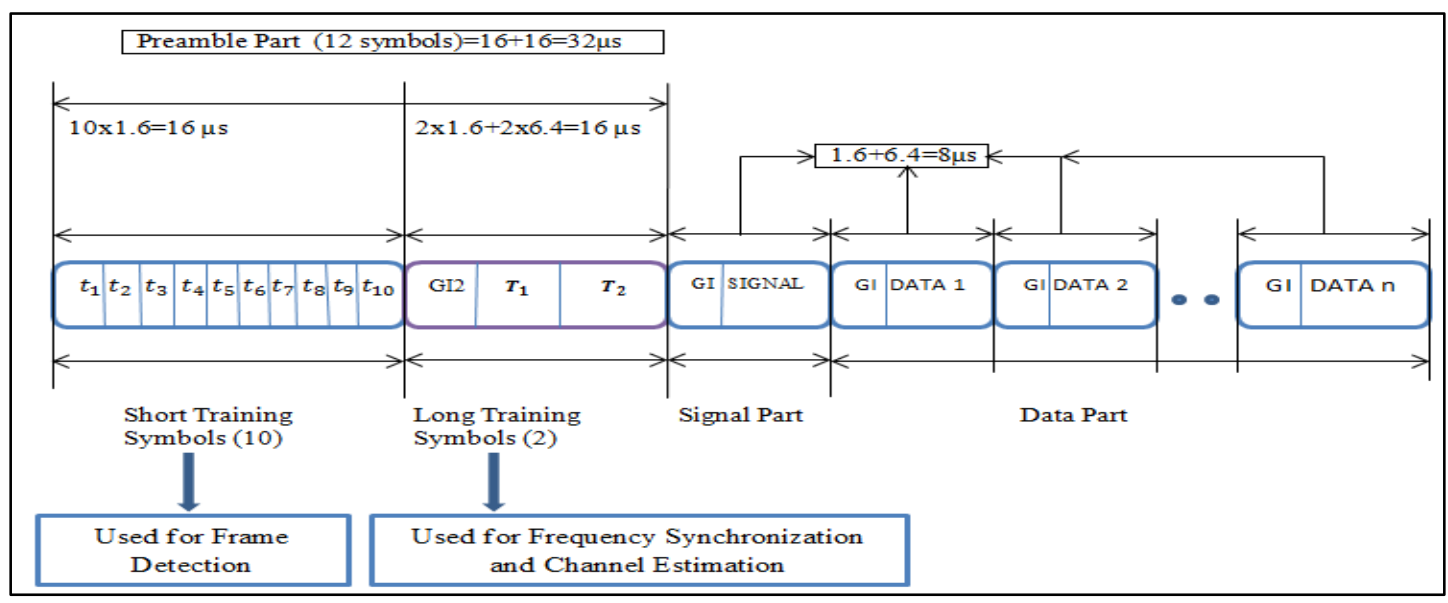

Fig. 5. IEEE 802.11p $10 \mathrm{MHz}$ PLCP Frame Contents

Table 2. IEEE 802.11 a/g/p wireless standard parameters and values [5], [23]

\begin{tabular}{|c|c|c|c|}
\hline Parameter & Notation & $\begin{array}{l}\text { IEEE 802.11a/g } \\
\text { Values }\end{array}$ & $\begin{array}{l}\text { IEEE 802.11p } \\
\text { values }\end{array}$ \\
\hline $\begin{array}{l}\text { Total OFDM Sub- } \\
\text { carrier }\end{array}$ & $N_{S C}$ & 64 & 64 \\
\hline Pilot Sub-carrier & $N_{P}$ & 4 & 4 \\
\hline Data Sub-carrier & $N_{D S C}$ & 48 & 48 \\
\hline Preamble Duration & & $16 \mu \mathrm{s}$ & $32 \mu \mathrm{s}$ \\
\hline Guard Time & GI & $0.8 \mu \mathrm{s}$ & $1.6 \mu \mathrm{s}$ \\
\hline Bit Rate (Mbps) & & $\begin{array}{l}\text { 6,9,12,18,24,26,48, } \\
54\end{array}$ & $\begin{array}{l}3,4.5,6,9,12,18,24 \\
, 27\end{array}$ \\
\hline Modulation Type & & $\begin{array}{l}\text { BPSK, QPSK, } \\
\text { 16/64-QAM }\end{array}$ & $\begin{array}{l}\text { BPSK, QPSK, } \\
\text { 16/64-QAM }\end{array}$ \\
\hline Bandwidth (MHz) & $\mathrm{B}$ & $20 \mathrm{MHz}$ & $10 \mathrm{MHz}$ \\
\hline Symbol Period & $\begin{array}{l}T_{\text {OFDM }} \\
=\frac{N_{S C}+G}{B}\end{array}$ & $4 \mu \mathrm{s}$ & $8 \mu \mathrm{s}$ \\
\hline $\begin{array}{l}\text { Sub-carrier } \\
\text { Frequency Spacing }\end{array}$ & $\Delta f=\frac{B}{N_{S C}}$ & $0.3125 \mathrm{MHz}$ & $0.15625 \mathrm{MHz}$ \\
\hline
\end{tabular}

Fig. 5 describes the packet preamble structures of IEEE 802.11p. Each IEEE $802.11 \mathrm{a} / \mathrm{g} / \mathrm{p}$ frames consists of preamble including 10 short training symbols $\left(t_{1}\right.$ to $\left.t_{10}\right)$ of each $1.6 \mu \mathrm{s}$ located at the beginning of each frame used to detect starting of a frame, 2 long training symbols ( $T_{1}$ and $T_{2}$ ) of each $6.4 \mu \mathrm{s}$ used for channel equalization and channel estimation. The rest of the frame is the signal field and data the field. Signal field contains the information about modulation schemes, coding rates etc. by using only one OFDM symbol and the data field generally used for transmitting data. Guard interval is used to avoid inter symbol interference. The frame detection algorithm is based on the autocorrelation of the short training sequence which is 16 samples repeating 10 times. The receiver feats this cyclic pattern of short training symbols and calculates the autocorrelation value $a$ of the incoming data sample stream $s$ with lag 16 by summing up the autocorrelation coefficients over an adjustable window $N_{\text {win }}$ [2]. 


$$
a(n)=\sum_{k=0}^{N_{\text {win }}-1} s(n+k) \bar{s}(n+k+16)
$$

Here $\bar{s}$ express the complex conjugate of $s$. Experimental result showed that adjustable window $N_{\text {win }}=48$ gives better performance and the summation over this window turns into moving average which acts as a low pass filter. The autocorrelation is high at the start of each IEEE $802.11 \mathrm{a} / \mathrm{g} / \mathrm{p}$ frame due to the cyclic property of short training sequence. The autocorrelation $a(n)$ is normalized with the average power $p(n)$ such that the receiver was independent of the absolute level of incoming samples [2].

$$
p(n)=\sum_{k=0}^{N_{\text {win }}-1} s(n+k) \bar{s}(n+k)
$$

Finally, the normalized autocorrelation coefficient $c$ can be expressed as [2]:

$$
c(n)=\frac{|a(n)|}{p(n)}
$$

Here, $|a(n)|$ denotes the magnitude of $a(n)$. Here the Fig. 6 given below expressed the IEEE 802.11 standard frame receiver schematics at GNU Radio companion. These schematics have basically 2 parts one is frame detection and another part is frame decoding.

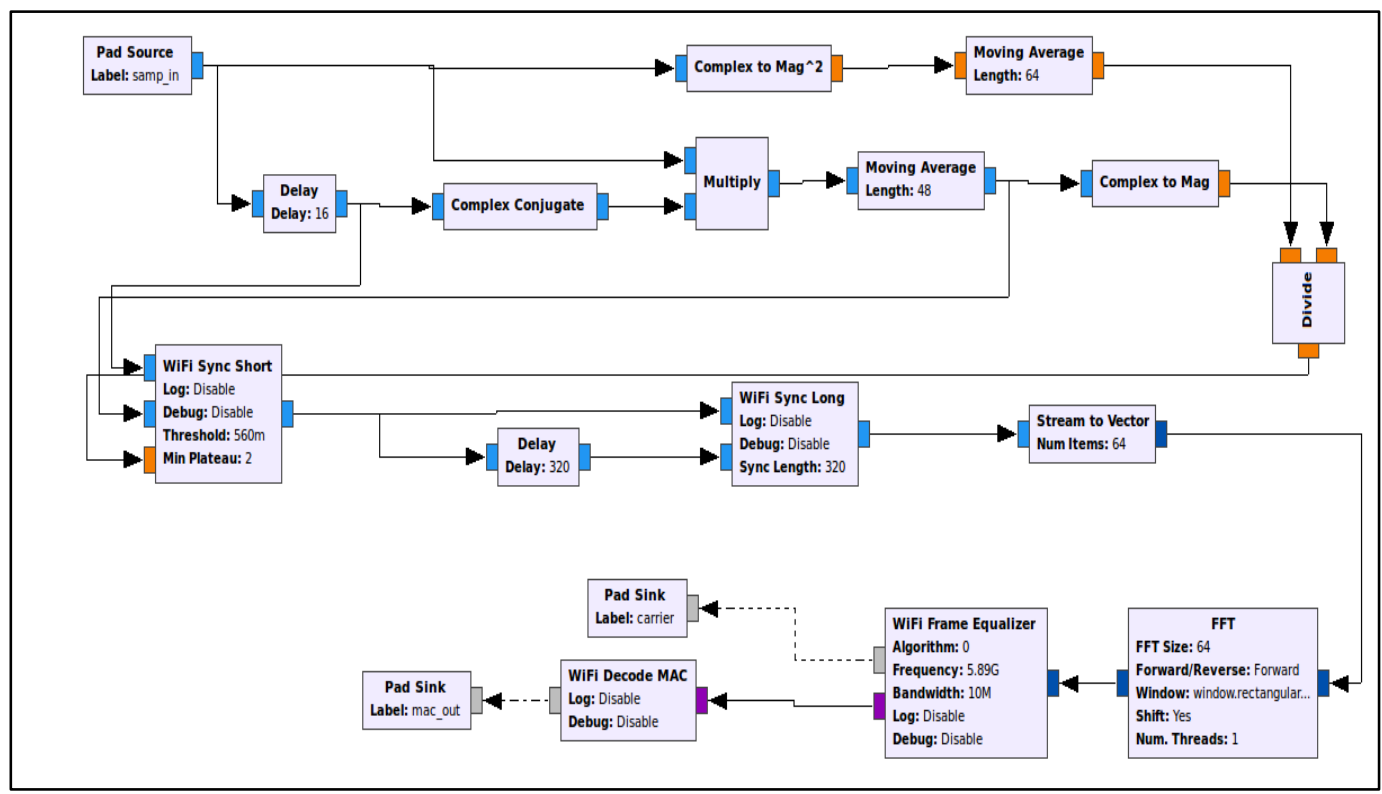

Fig. 6. IEEE 802.11 standard packet receiver at GNU Radio Companion [22]

After that, normalized autocorrelation coefficients $c(n)$ and the received samples from the USRP are sent to the WiFi Sync Short Block as input samples which act as like as a valve. WiFi Sync Long block is used for frequency offset correction and symbol alignment. The transmitter and receiver oscillators are clocking at slightly different frequencies due to this there will be frequency offset between two devices. For the proper OFDM transmission, the transmitter and receiver must be synchronized with the same clocking. The short training 
preambles are used to estimate the frequency offset between. Let us consider $s(n)$ represents a sample of the short preamble and $s(n)$ corresponds to the sample $s(n+16)$ due to its cyclic property. The frequency offset $d f$ can be expressed as the average of the argument of the product $s(n) \bar{s}(n+16)$ for all the 16 samples which are given below [2]:

$$
d f=\frac{1}{16} \arg \left\{\sum_{n=0}^{N_{\text {short }}-1-16} s(n) \bar{s}(n+16)\right\}
$$

Here $N_{\text {short }}$ is the length of the short training samples. The calculated frequency offset $d f$ is applied to every long training preamble and data symbols as well to get the perfect synchronization. In the next block, the time domain samples are converted to the frequency domain using FFT block. After the FFT block data samples are sent to OFDM Equalize Symbol block for the channel estimation and phase offset correction. The Phase offset is introduced between the transmitter and receiver as the sampling time is not synchronized properly and symbol alignment in the receiver is not correct. Linear regression method is used to estimate the phase offset between OFDM samples and it is linear with the frequency. Let us consider, there are a set of $\mathrm{N}$ order pairs $\left(x_{0}, y_{0}\right),\left(x_{1}, y_{1}\right),\left(x_{2}, y_{2}\right) \ldots \ldots \ldots,\left(x_{N-1}\right.$, $\left.y_{N-1}\right)$ and through this points using LS method, a regression line $\hat{y}=a+b \hat{x}$ is computed. IEEE a/g/p defines 4 pilot sub-carriers which are used to compute the values of $a$ and $b$ to equalize the phase offset of the OFDM data symbols in the physical layer frame. The final stage of the receiver is the decoding of the payload using WiFi Decode Mac block which performed the demodulation, de-interleaving, convolution decoding, puncturing and descrambling [1]-[3] etc.

\section{Wireless Channel Models}

Multipath causes the propagation of radio signals transmitted from one antenna to another through two or more paths. In urban areas, fading arises due to the different height of the transmitting and receiving antennas as there is no particular line of sight (NLOS) to propagate the signal from one antenna to another [11]. But fading may happen even there is a presence of a Line of Sight (LOS) due to the reflection, scattering etc. of the transmitted signal from the ground and surrounding area objects. The receiving antenna will get a resultant signal which can vary widely in amplitude or even in phase based on the distribution of the intensity and the bandwidth of the transmitted signal. The amplitude variations of multipath fading signals are followed by Rayleigh and Rician distributions. Multipath propagation can be categorized as large scale and small scale fading. Small scale fading can be expressed as rapid fluctuations of the amplitude or phase of the transmitted radio signal over a very short period of time or short travel distance. Large scale fading is the consequence of signal attenuation due to the propagation over long distances and diffraction around large objects. Multipath small scale fading effect causes few rapid changes in radio channels media such as rapid changes in signal strength level, time dispersion produced by multipath propagation path delays and random frequency modulation due to varying Doppler shifts of different multipath signals [20]. The small scale fading can be expressed as a linear filter with the time varying impulse response of a wireless channel. Time variation is introduced due to the receiver motion in space and filtering nature is introduced due to the summation of amplitudes, delays of multiple arriving waves at any instant of time. However based on the time delay spread small scale fading can be categorized as flat fading and frequency selective fading. As per Doppler spread small scale fading can be categorized as 
fast fading and slow fading. In GNU Radio, the fading model blocks exist for both Rayleigh and Rician distributions. The models approximate a fading channel using the sum of sinusoids method (SOS) for the number of anticipated multipath components. Generally, the number of sinusoids value is set to 8 as it is considered a decent value. It takes other input parameters such as the normalized value of Doppler frequency shift, a line-of-sight (LOS) parameter which is false for Rayleigh distribution (NLOS) and true for Rician distribution (LOS), the Rician factor $\mathrm{K}$ and a random seed to the noise generators [21].
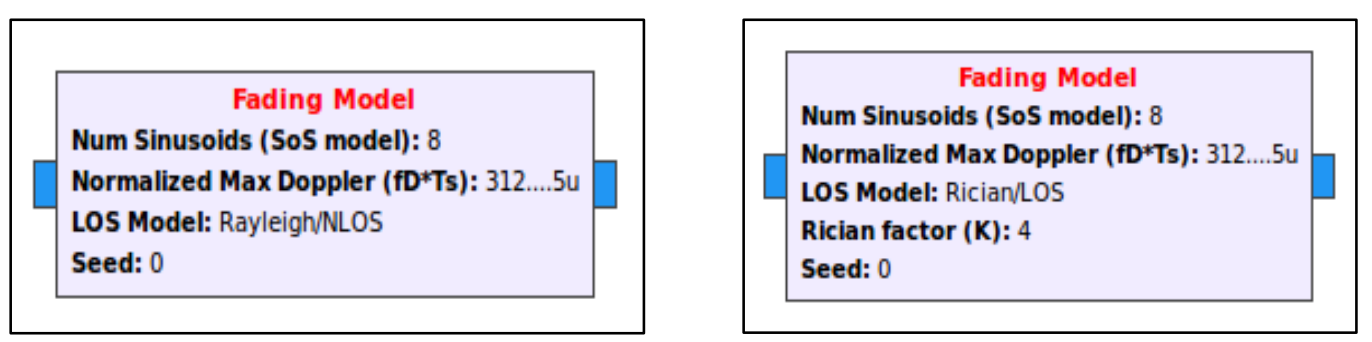

Fig. 7. The Rayleigh (NLOS) and Rician (LOS) model block at GNU Radio

Frequency selective fading model takes the input of power delay profile (PDP) to design a time varying multiple taps impulse response filtered channel model. In this fading model, PDP is given as a vector to provide the time delay of each impulse. The PDP magnitudes define the corresponding magnitudes to each time delay. A number of possible taps length of the filter is also assigned to simulate the channel fading behavior and to interpolate the power delay profile [21].

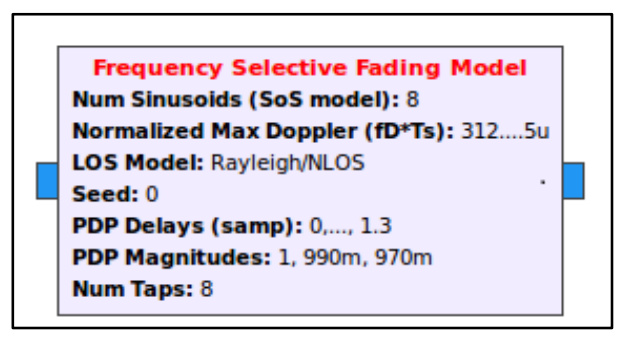

Fig. 8. Frequency Selective Fading Model block at GNU Radio

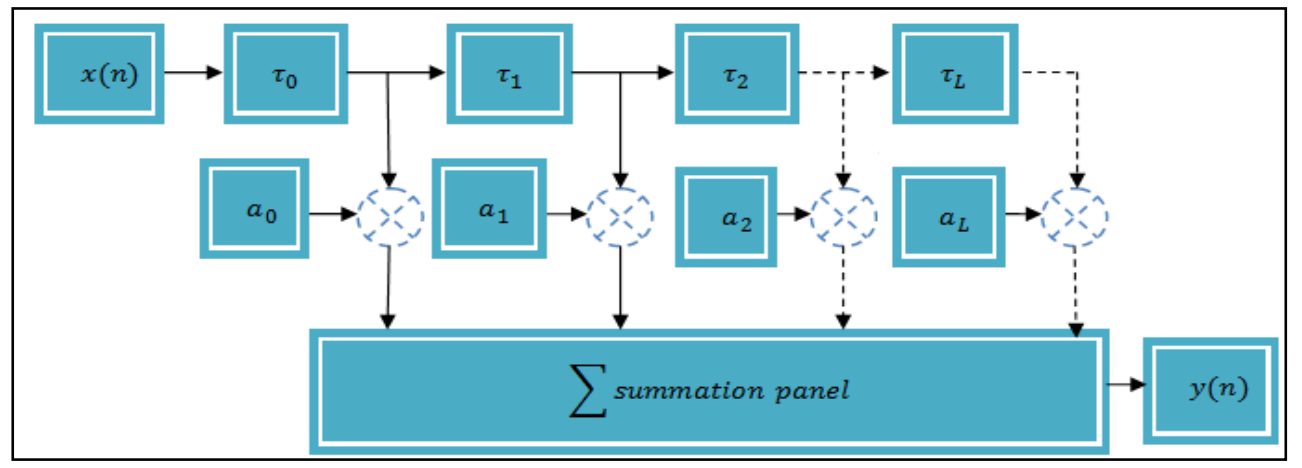

Fig. 9. L-tapped delay line FIR filter to describe the fading channel model characteristics. ( $\tau_{L}$ is the delay of each tap during propagation, $a_{L}$ is the tap gain which causes the magnitude variations of the impulses) 
Additive White Gaussian Noise (AWGN) is the random basic noise model that exists in nature. This noise spectrum is uniform for all frequencies and the amplitude variation follows Gaussian Probability Density Function (PDF) [12]. Due to this reason, it has a uniform frequency spectrum shown in Fig. 10 (b) and constellation diagram show the higher density at the center and less density outer from the center which is shown in Fig. 10 (c). Additions of this noise to a transmitted radio signal during propagation through the wireless causes the signal amplitude variations. The channel model simulates AWGN with a simple static multipath environment using some parameters such as frequency offset; timing offset using epsilon, taps of FIR filter to add delay to multipath and a random number generator seed for the noise source [21].

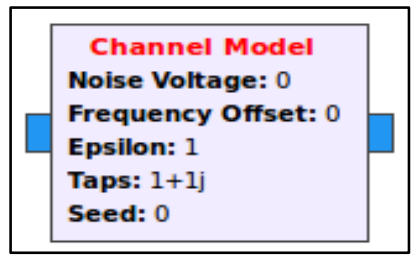

(a)

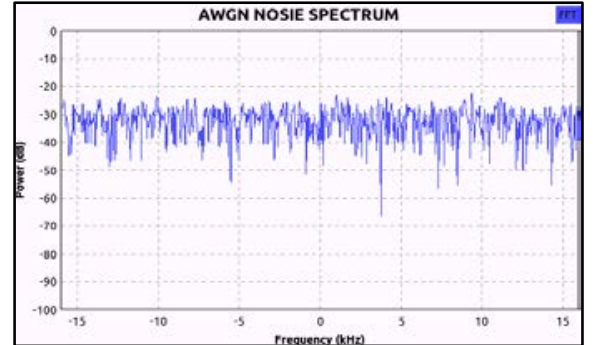

(b)

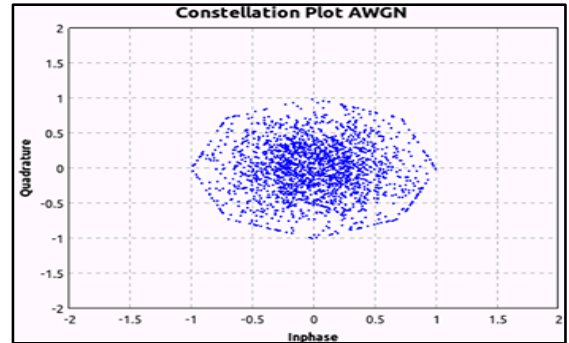

(c)

Fig. 10. (a) AWGN Channel Model Block at GNU Radio (b) AWGN noise spectrum (c) Constellation diagram of AWGN

\section{FER Calculation of IEEE $802.11 \mathrm{a} / \mathrm{g} / \mathrm{p}$ for AWGN Channel}

Generally, NIST and YANS error rate models are so much popular to analyze the performance of an IEEE 802.11 standard WLAN. These models compute SNR based on the parameters used in simulation model such as noise figure, noise floor, path loss model etc. The FER is computed based on the applied modulation scheme, coding rate, and frame size. Let us assume that convolution coding and hard decision Viterbi decoding are applied to the data symbols and transmitted over an AWGN channel model. In IEEE $802.11 \mathrm{a} / \mathrm{g} / \mathrm{p}$ the bit error probability is upper bound by following Chernoff Bound as per the NIST error rate model [18]:

$$
P_{b}<\frac{1}{k} \sum_{d=d_{\text {free }}}^{\infty} B_{d} P(d), P(d)=[4 p(1-p)]^{\frac{d}{2}}
$$

Here, $d_{\text {free }}$ is the free distance of the convolution code, multiplication factor $B_{d}$ is the total number of non-zero information bits that are in error while an incorrect path is chosen for the specified hamming distance $\mathrm{d}, P(d)$ is the probability of selecting a weight $d$ output 
sequence as the transmitted code sequence, $p$ is the simply the un-coded BER and $k$ is the number of information bits per clock cycle. The signal to noise power ratio (SNR) in AWGN is estimated using the equation given below [12]:

$$
S N R[d B]=P_{\text {rec }}-N_{F}-N_{1}
$$

Here, $P_{r e c}$ is the received signal power at the receiving antenna, $N_{F}$ is the noise figure and $N_{1}$ is the thermal noise floor. YANS model estimated the ratio between the energy per bit $\left(E_{b}\right)$ and noise power spectral density $\left(N_{0}\right)$ as $\frac{\mathrm{E}_{\mathrm{b}}}{\mathrm{N}_{\mathrm{o}}}=\operatorname{SNR} \frac{\mathrm{B}_{\mathrm{t}}}{\mathrm{R}_{\mathrm{b}}}$, here $B_{t}$ is the noise bandwidth and $R_{b}$ is the raw bit rate calculated by the number of bits per OFDM symbol over the symbol interval time. This model does not account for the reduction of energy due to the cyclic prefix (CP) and the reduction of the net energy due to the pilot carriers which are basically not used for carrying information. On the other hand, NIST model estimated $\frac{E_{b}}{N_{0}}=\frac{S N R}{\log _{2}(M)}$, where $\mathrm{M}=$ number of signal constellations. NIST model does not account for the ratio of used sub-carriers in OFDM system and CP. However, in order to calculate more accurate, BER and FER for more precise estimation of $\frac{E_{b}}{N_{0}}$ are required which given below [12]:

$$
\frac{E_{b}}{N_{0}}=S N R\left(\frac{N_{F F T}}{N_{D C}+N_{P C}}\right) \times\left(\frac{1}{N_{C B P S}}\right)
$$

Here $N_{F F T}$ is the FFT length, $N_{D C}$ is the number of data sub-carriers, $N_{P C}$ is the number of pilot carriers and $N_{C B P S}$ is the number of coded bit per symbol in each OFDM sub-carrier. The FER can be expressed as $F E R=1-\left(1-P_{b}\right)^{L}$; Here $\mathrm{L}$ is the aggregated MAC frame size. The standard size of L is basically 441 bytes. BER for the M-PSK modulated signal in AWGN channel can be expressed as given below [24]:

$$
P_{M-P S K}=\frac{2}{\max \left(\log _{2} M, 2\right)} \sum_{k=1}^{\max (M / 4,1)} Q\left(\sqrt{\frac{2 E_{b} \log _{2} M}{N_{0}}} \sin \frac{(2 k-1) \pi}{M}\right)
$$

For $\mathrm{M}=2$, the above equation reduces to BER of BPSK modulation $P_{B P S K}=Q\left(\sqrt{\frac{2 E_{b}}{N_{0}}}\right)$, here the error function $\mathrm{Q}$ is expressed as given below:

$$
Q(x)=\frac{1}{2 \pi} \int_{x}^{\infty} \exp \left(-\frac{t^{2}}{2}\right) d t=\frac{1}{2} \operatorname{erfc}\left(\frac{x}{\sqrt{2}}\right)
$$

BER for the M-QAM modulated signal in AWGN channel can be expressed as given below [24]:

$$
P_{M-Q A M}=\frac{4}{\log _{2} M} Q\left[\sqrt{\frac{3\left(\log _{2} M\right) E_{b}}{(M-1) N_{0}}}\right]
$$

To estimate the performance of the receiver FER is calculated for different values of SNR (dB) expressed in terms of $\frac{E_{b}}{N_{0}}$. The data is transmitted over AWGN channel by choosing 16QAM modulation scheme for each sub-carrier with rate 24 Mbps. The outputs of the simulated model at GNU Radio during real-time data transmission over AWGN channel 
using USRP devices are given in Fig. 11. According to the above equations no. (15) and (16), both Bit Error Rate (BER) and FER are the functions of SNR and $\frac{E_{b}}{N_{0}}$. As a result, with decreasing SNR (dB) the coded and mapped constellations points that are carrying the data symbols mentioned at Fig. 11 \{(a), (b)\} are spreading from the center and almost try to overlap with each other due to the effect of AWGN. It causes the ISI and affects to maintain the orthogonal property between the OFDM sub-carriers. To reduce the FER channel estimation and equalization should be investigated at the receiver end.

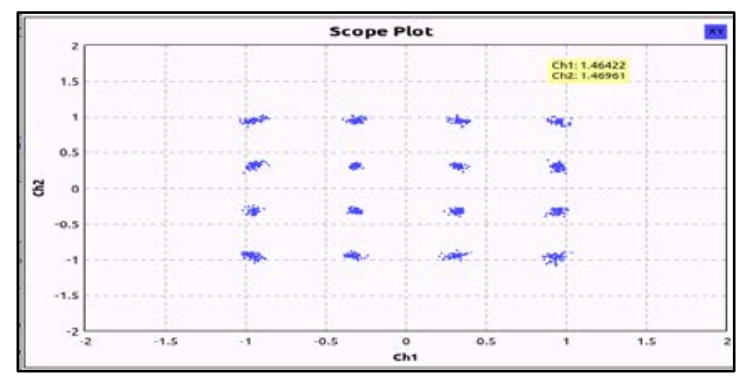

(a) $\mathrm{SNR}=30 \mathrm{~dB}$

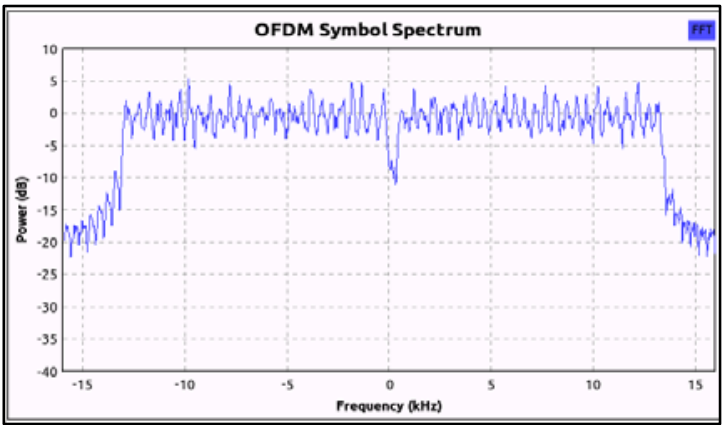

(c) Power $(\mathrm{dB})$ vs. Frequency $(\mathrm{kHz}), \mathrm{SNR}=30 \mathrm{~dB}$

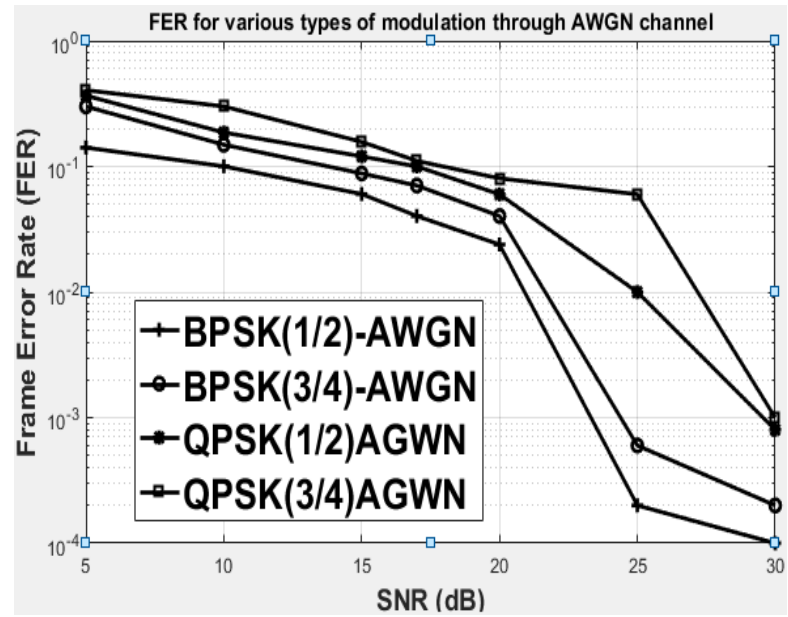

(e) FER vs. SNR, M-PSK

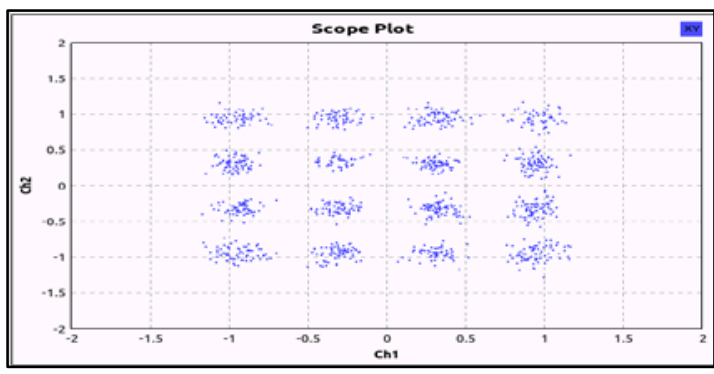

(b) $\mathrm{SNR}=20 \mathrm{~dB}$

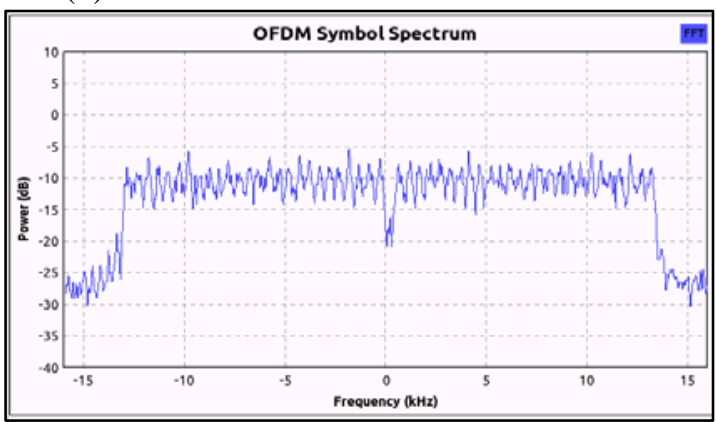

(d) Power (dB) vs. Frequency $(\mathrm{kHz}), \mathrm{SNR}=20 \mathrm{~dB}$

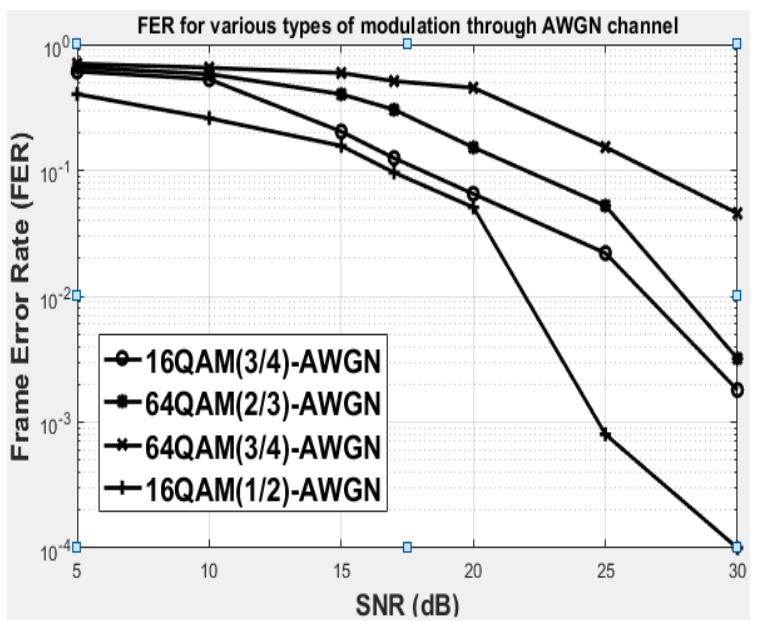

(f) FER vs. SNR, M-QAM

Fig. 11. [(a), (b)] 16-QAM (1/2) constellation points for $S N R=30 \mathrm{~dB}$ and $20 \mathrm{~dB}$ respectively with 24 Mbps data rate; [(c), (d)] OFDM spectrum for SNR $=30 \mathrm{~dB}$ and $20 \mathrm{~dB}$ respectively; [(e), (f)] FER performance of OFDM for $M \in\{2,4\}$-PSK and $M \in\{16,64\}$-QAM with different coding rate $\in\{1 / 2$, 


\section{FER Calculation of IEEE $802.11 \mathrm{a} / \mathrm{g} / \mathrm{p}$ for Fading Wireless Channel}

Mobile wireless channel considered as the flat fading Rayleigh channel with Jake spectrum. A particular frame will be successfully transmitted when it is fully in the inter-fading state. Let us consider some random variable given below: $t_{i}=$ inter fading duration, $T_{i}$ is the mean value of $t_{i}, t_{f}=$ fading duration, $T_{f}$ is the mean value of $t_{f}, t_{F D}=$ frame duration and $T_{F D}$ is the mean value of $t_{F D}$. A particular threshold value of the received signal power is used to determine the frame either is in the fading state or inter fading state. If any part of the frame is in the fading state, then the frame delivery will be unsuccessful. The threshold is known as fading margin $m=\frac{R_{r e q}}{R_{r m s}}$, here $R_{r e q}$ is the required received power level and $R_{r m s}$ is the mean received power level. FER can be written as [11]:

$$
F E R=1-\frac{T_{i}}{T_{i}+T_{f}} P\left(t_{i}>T_{F D}\right)
$$

For both $t_{i}$ and $t_{f}$, exponential distributions are assumed and the probability of $P\left(t_{i}>T_{F D}\right)$ can be written as $e^{-\frac{T_{F D}}{T_{i}}}$.FER in Rayleigh fading channel can be expressed as given below [11]:

$$
F E R=1-e^{\left(-m-f_{m} \sqrt{2 \pi m} T_{F D}\right)}
$$

$f_{m}$ is the maximum Doppler frequency, defined as $f_{m}=\frac{v}{\lambda}=\frac{f_{c} v}{c}$. Here, $v=$ velocity of the mobile $\left(\mathrm{ms}^{-1}\right), f_{c}=$ carrier frequency $5.89 \mathrm{GHz}$ and $c=3 \times 10^{8} \mathrm{~ms}^{-1}$. Normalized Doppler frequency can be defined as $f_{D}=f_{m} T_{s}$, here $T_{s}$ is the symbol period. As a result, FER in wireless channel depends on Doppler frequency shift, fading margin and frame duration. Again to estimate the performance of the receiver, FER is calculated for different values of SNR (dB) where the selected data rate is 24 Mbps, the chosen modulation scheme is 16QAM and transmission medium is the frequency selective fading channel. The channel is simulated by introducing a tapped delay line FIR filter with an exponentially decaying average power delay profile (PDP). The number of taps given in FIR filter is 8 and tap characteristics is chosen as Rayleigh distributed (NLOS) and Rician distributed (LOS) exclusively. A particular normalized Doppler shift $\left(f_{D} T_{S}\right)$ is added to get the relative characteristics while the receiver is not stationary. In case of Rician distribution, the transmitted signal experienced a dominant nonfading signal component due to the presence of a line of sight (LOS) path between the transceiver. In this context, many weaker random multipath signals arriving at different angles produced by reflection, diffraction or scattering effect are superimposed on the dominant signal. The ratio between the deterministic signal power and the variance of multipath known as the Rician factor $K$ is set equal to 4 during simulation.

The outputs of the simulated model at GRC during real-time data transmission over AWGN and frequency selective fading channel model (Rayleigh Distributed-NLOS, Rician Distributed- LOS) using USRP devices are given respectively at Fig. 12, 13. The constellations diagrams at Fig. 12 and 13 \{(a), (b)\} shows that for the increasing Doppler shifts the coded and mapped information data symbols carried by the constellations points of 16-QAM are spreading and overlapping to each other. The OFDM signal spectrums are also 
losing the ideal shapes meaning that the orthogonal property between multiple sub-carriers are affected. Therefore, proper channel estimation is required for the error rate and ISI minimization. The FER vs. SNR (dB) curves mentioned at Fig. 12 and 13 showed comparatively less FER for less puncturing rate $\{$ M-PSK (1/2), M-QAM (1/2) $\}$ as well as for the lower data rate.

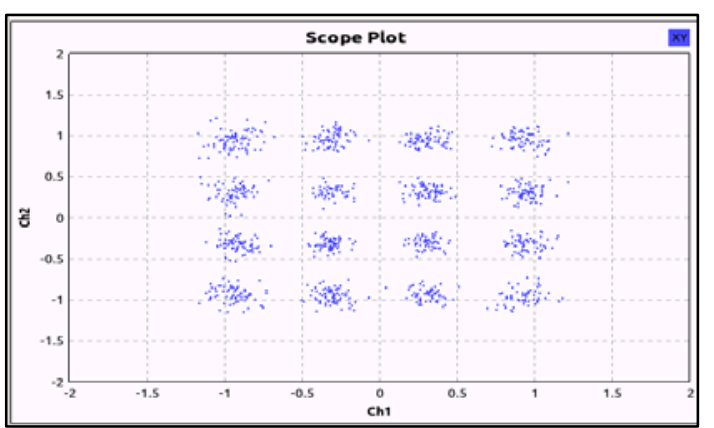

(a) $\mathrm{SNR}=20 \mathrm{~dB}, f_{d} T_{s}=0.0015$

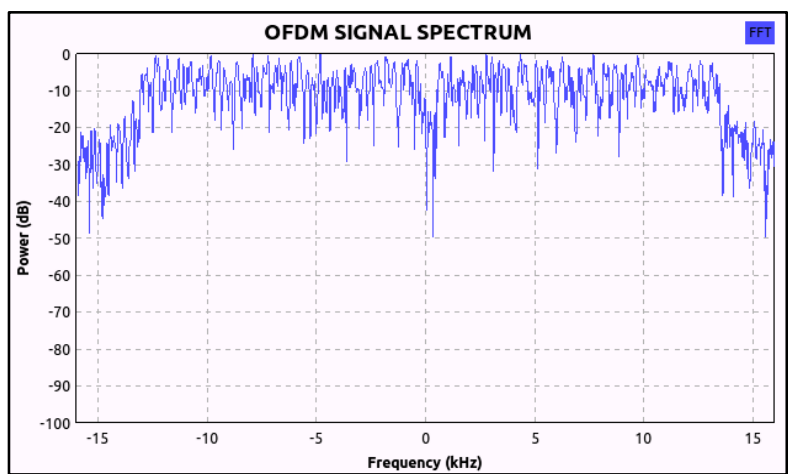

(c) Power (dB) vs. Frequency (kHz), SNR=20 dB, $f_{d} T_{s}=0.0015$

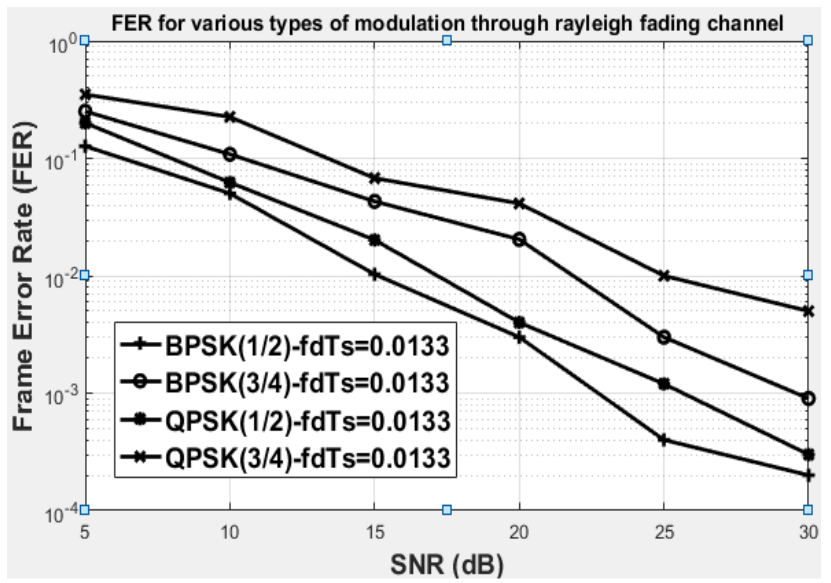

(e) FER vs. SNR, M-PSK, $f_{d} T_{s}=0.0133$

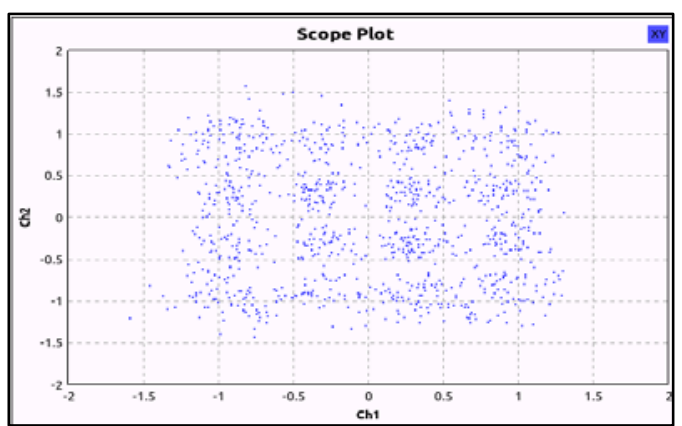

(b) $\mathrm{SNR}=20 \mathrm{~dB}, f_{d} T_{s}=0.0133$

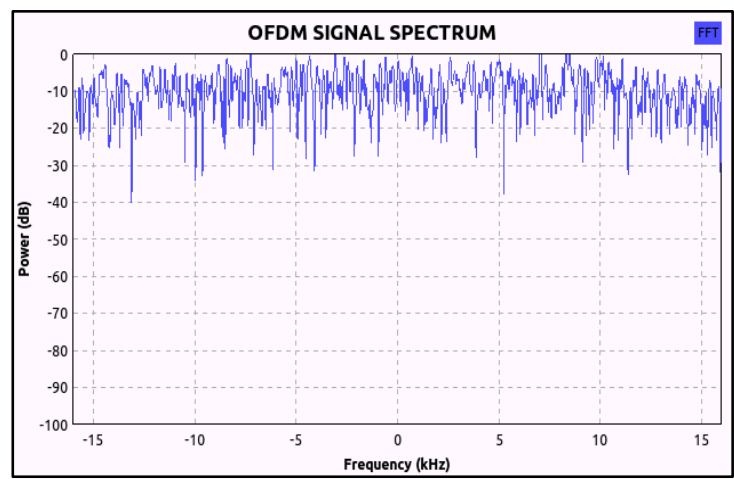

(d) Power (dB) vs. Frequency (kHz), SNR $=20 \mathrm{~dB}, f_{d} T_{s}=0.0133$

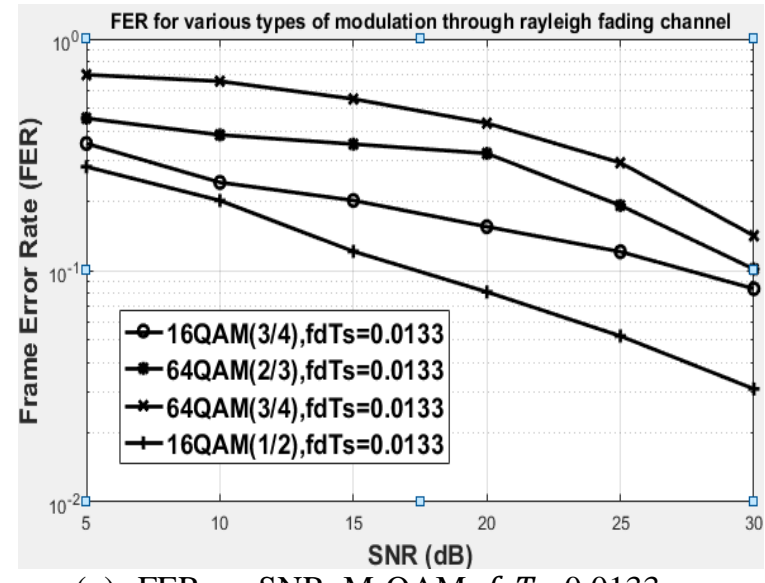

(g) FER vs. SNR, M-QAM, $f_{d} T_{s}=0.0133$

Fig. 12. [(a), (b)] 16-QAM Constellation Points [(c), (d)] OFDM symbol spectrum [for Rayleigh distributed frequency selective fading channel having $\mathrm{SNR}=20 \mathrm{~dB}$ and $f_{d} T_{s}=0.0015,0.0133$ respectively] [(e), (f)] FER performance of OFDM for $M \in\{2,4\}$-PSK and $M \in\{16,64\}$-QAM with different coding rate $\in\{1 / 2,2 / 3,3 / 4\}, f_{d} T_{s}=0.0133$ 


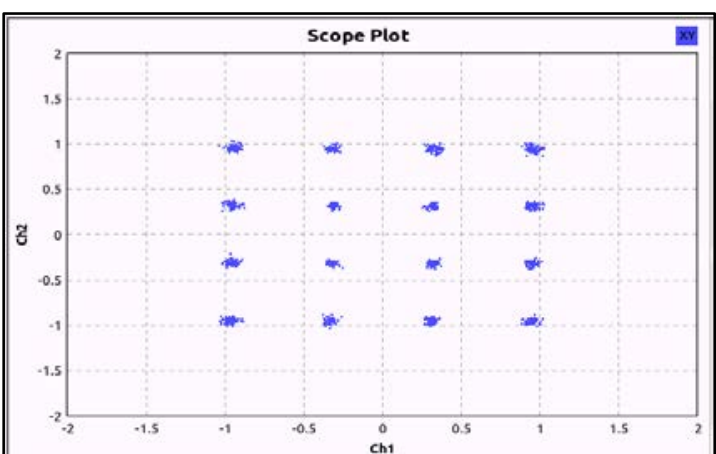

(a) $\mathrm{SNR}=20 \mathrm{~dB}, f_{d} T_{s}=0.0015$

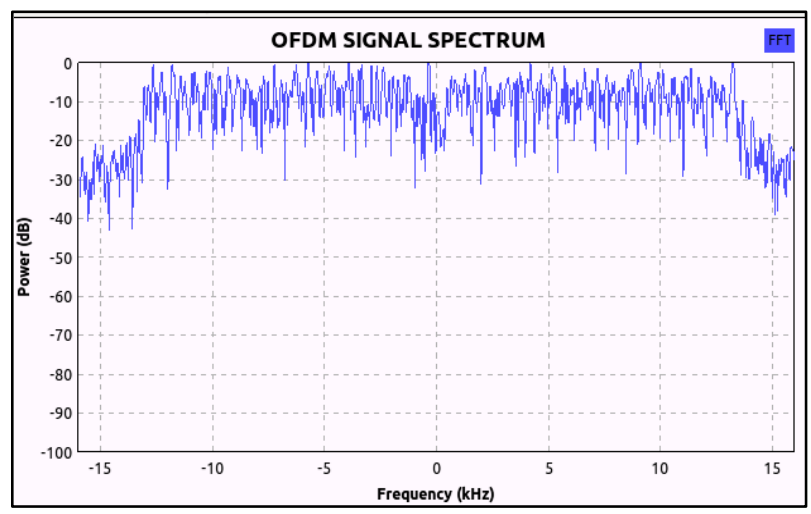

(c) Power (dB) vs. Frequency (kHz), SNR=20dB, $f_{d} T_{s}=0.0015$

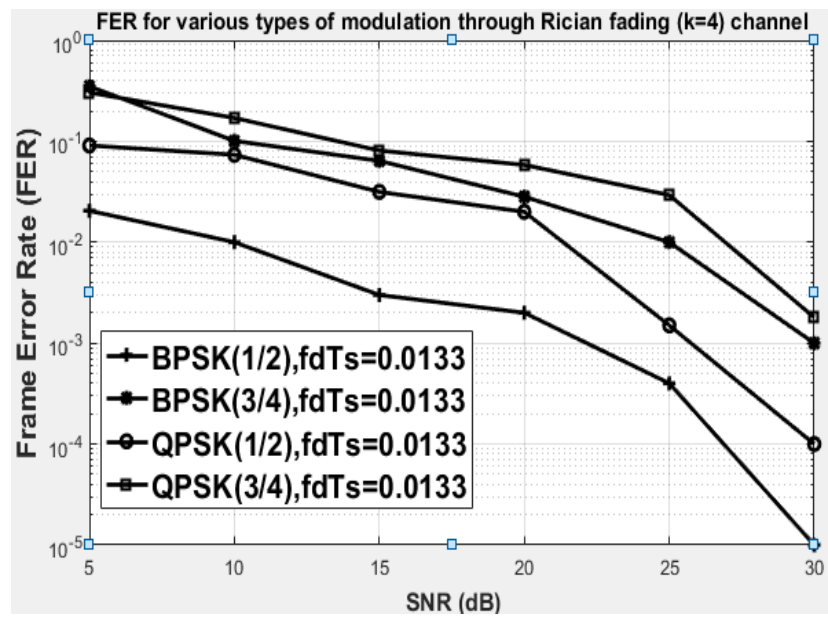

(e) FER vs. SNR, M-PSK, $f_{d} T_{s}=0.0133$

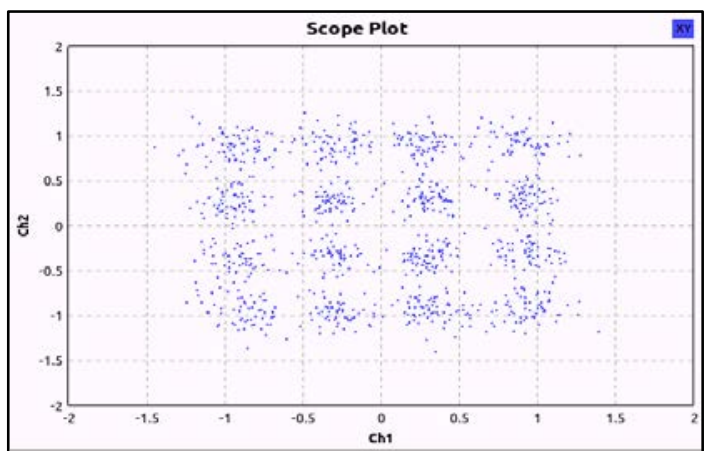

(b) $\mathrm{SNR}=20 \mathrm{~dB}, f_{d} T_{s}=0.0133$

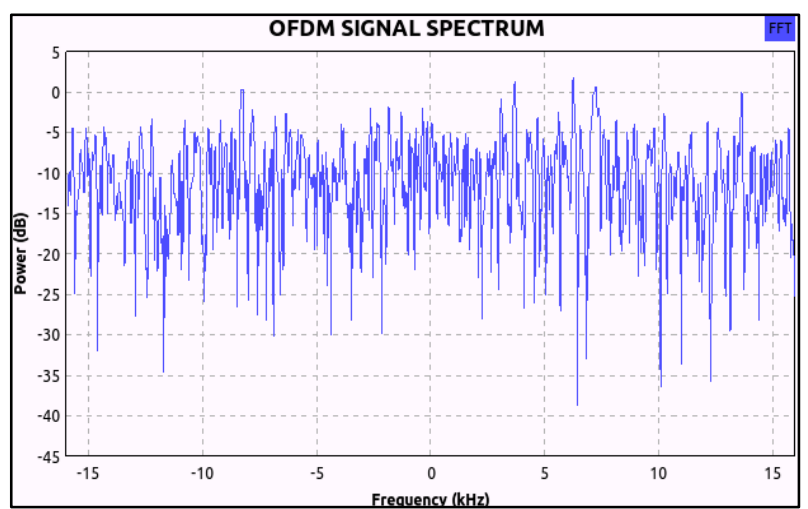

(d) Power (dB) vs. Frequency (kHz), $\mathrm{SNR}=20 \mathrm{~dB}, f_{d} T_{s}=0.0133$

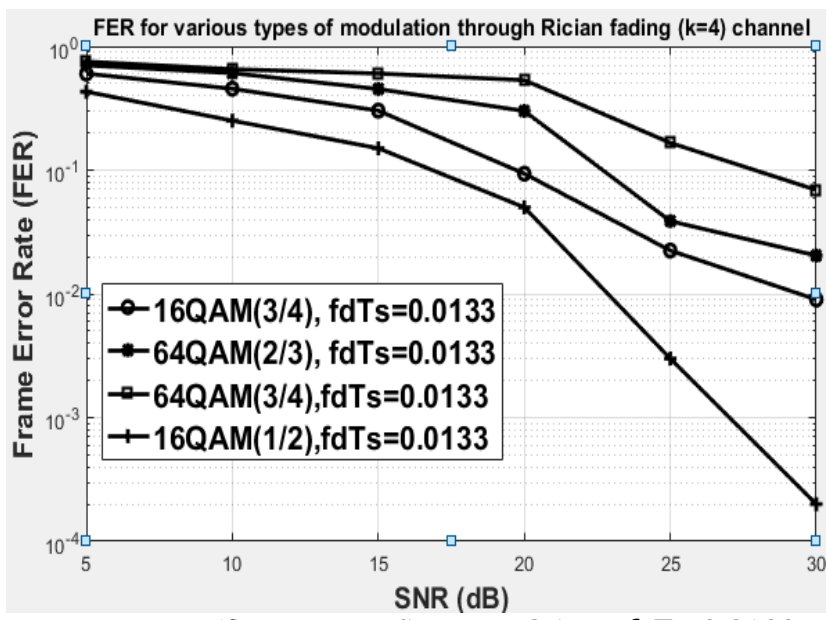

(f) FER vs. SNR, M-QAM, $f_{d} T_{s}=0.0133$

Fig. 13. [(a), (b)] 16-QAM Constellation Points [(c), (d)] OFDM symbol spectrum [for Rician distributed frequency selective fading channel having $S N R=20 \mathrm{~dB}$, Rician Factor $\mathrm{K}=4$ and normalized Doppler frequency $f_{d} T_{s}=0.0015,0.0133$ respectively] [(e), (f)] FER performance of OFDM for $M \in\{2,4\}$-PSK and $M \in\{16,64\}$-QAM with different coding rate $\in\{1 / 2,2 / 3,3 / 4\}$, $f_{d} T_{s}=0.0133$ 


\section{Channel Estimation (CE) Techniques}

The CE process is the approximation and characterizing the effect of physical medium on the transmitted data symbols. It allows the implementation of coherent demodulation technique. CE technique basically uses the pilot reference signals of OFDM to estimate the channel characteristics. This technique minimizes the Mean Square Error (MSE) in order to get more accurate data symbols at the receiver end by utilizing the less computational method. Based on the help of the position of neighboring pilot sub-carries both time and frequency-domain interpolation are required to get the $\mathrm{CE}$ of each data sub-carrier in time-frequency plane as the pilots are assigned combined block-comb allocation method. If the receiver has the prior information of the transmitting data over the channel, it can utilize that knowledge in the estimation process to get the more accurate data. Predefined training symbols are used at the beginning of each IEEE 802.11 standard WLAN data packets with safety guard interval to get the convergence of the estimator. IEEE $802.11 \mathrm{a} / \mathrm{g} / \mathrm{p}$ uses two long preambles $T_{1}$ and $T_{2}$ in the Least Square (LS) CE process. Let us consider for each OFDM frame the received long preambles of a particular OFDM symbol are denoted as $Y_{T_{1}}$ and $Y_{T_{2}}$ and predefined frequency domain long training symbols are $X_{T_{1}}$ and $X_{T_{2}}$. Then the LS CE transfer function is expressed as given below [3], [8]:

$$
\widetilde{H_{L S}}(k)=\frac{1}{2}\left(\frac{Y_{T_{1}}}{X_{T_{1}}}+\frac{Y_{T_{2}}}{X_{T_{2}}}\right)
$$

Above estimation is used to equalize the rest of the OFDM symbols. LS CE algorithm is expressed as the ratio between the output $Y(k)$ and input data $X(k)$ sequence. The LS estimation of the channel transfer function $\widetilde{H_{L S}}(k)$ can be written as given below [9], [17]:

$$
\begin{aligned}
\widetilde{H_{L S}}(k)=\frac{Y(k)}{X(k)}= & Y(k) X^{-1}(k)=\left[\frac{y_{0}}{x_{0}}, \frac{y_{1}}{x_{1}}, \ldots ., \frac{y_{N-1}}{x_{N-1}}\right]^{T}=\frac{X(k) H(k)+W(k)}{X(k)} \\
& =H(k)+\frac{W(k)}{X(k)} \quad(22)
\end{aligned}
$$

The LE CE of the channel transfer function at pilot positions can be expressed as:

$$
\begin{aligned}
\widetilde{H}_{L S, P}\left(k_{p}\right)=\frac{Y_{P}\left(k_{p}\right)}{X_{P}\left(k_{p}\right)}=X_{P}^{-1}\left(k_{p}\right) Y_{P}\left(k_{p}\right) \\
=\left[\frac{Y_{P}(0)}{X_{P}(0)} \frac{Y_{P}(1)}{X_{P}(1)}, \ldots \ldots \ldots, \frac{Y_{P}\left(N_{P}-1\right)}{X_{P}\left(N_{P}-1\right)}\right]^{T}
\end{aligned}
$$

Here, a number of pilot signal $N_{P}=4, Y_{P}\left(k_{p}\right)$ is the received signal in the pilot positions (21,-7, 7, 21) and $X_{P}\left(k_{p}\right)$ is the known transmitted pilot signal. The LS pilot estimated transfer function $\widetilde{H}_{L S, P}\left(k_{p}\right)$ is used to determine the channel estimate transfer function at each neighboring sub-carrier. As the pilots are assigned in both time and frequency domain, therefore, 2-D channel interpolation is required to compute the channel transfer function at each sub-carrier $\widetilde{H_{L S}}(k)$ from $\widetilde{H}_{L S, P}\left(k_{p}\right)$ that can be expressed as [8]:

$$
\widetilde{H_{L S}}(k)=\sum_{n_{p} k_{p}} F\left[(n, k) ;\left(n_{p}, k_{p}\right)\right] \cdot \widetilde{H}_{L S, P}\left(k_{p}\right)
$$


Here $F\left[(n, k) ;\left(n_{p}, k_{p}\right)\right]$ is the estimation filter which coefficients are needed to be estimated to get the estimation at a particular OFDM sub-carrier. The term $(n, k)$ defines the position of particular OFDM channel that has to be estimated, $n$ denotes the OFDM symbol and $k$ is the sub-carrier index. The filter coefficients can be estimated by using a low-pass filter interpolation (LPI) technique at both time and frequency domain.

A signal $x(k)$ having bandwidth B can be restored properly from its samples $x(n)$ using an ideal low pass filter with bandwidth $W=\frac{F_{S}}{2}$ only if the sampling frequency $F_{S} \geq 2 B$. The channel transfer function $\widetilde{H_{L S}}(k)$ is sampled with the rate $f_{s, t i m e}=\frac{F_{S R}}{f_{t}}$, where $F_{S R}$ is the symbol rate (symbols/sec) and $f_{t}$ is the pilot separation along with time domain. The channel maximum Doppler frequency $f_{m}$ defines the rate of change of the channel across the time direction. A low-pass filter can be used for the time-domain interpolation by avoiding aliasing only when $f_{m} \leq \frac{F_{S R}}{2 f_{t}}$. For the frequency domain interpolation channel transfer function $\widetilde{H_{L S}}(k)$ is sampled with the frequency $f_{s, f r e q}=f_{f} . \Delta f$, where $f_{f}$ is the pilot's separation along with frequency domain, $\Delta f$ is the sub-carrier spacing. The time period is calculated from the sampling time $\frac{1}{f_{f} \cdot \Delta f}=\frac{T_{S W C P}}{f_{f}}$, where $T_{S W C P}$ is the OFDM symbol period without the cyclic prefix; it gives the periodic replicas of the channel impulse response $h(n, \tau)$.

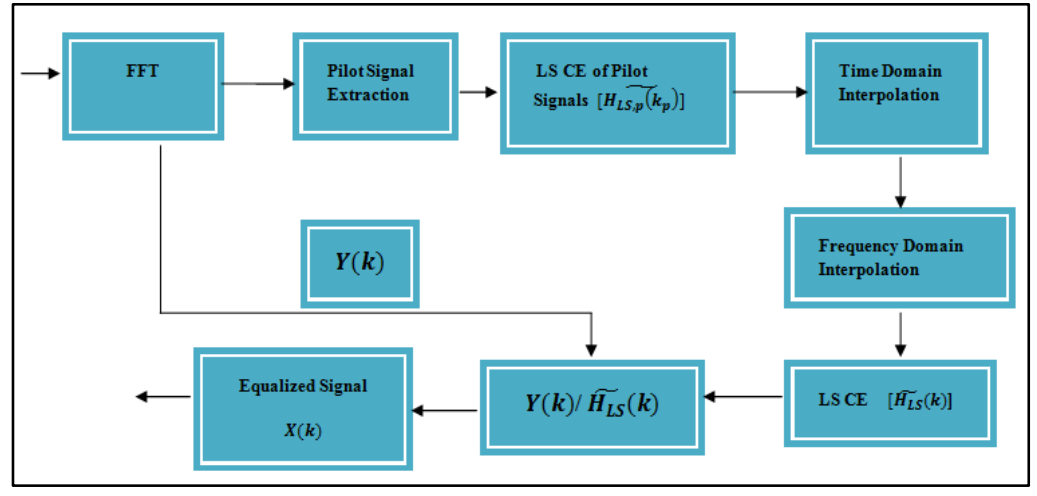

Fig. 14. LS CE from pilot signal using LPI technique

Therefore, a low pass filter can be used along with frequency domain for channel interpolation without aliasing only when maximum delay spread of the channel $\tau_{\max } \leq$ $\frac{T_{S W C P}}{f_{f}}$. Fig. 15 gives the FER performance comparisons after applying LS CE technique during data symbol transmission over AWGN and Rayleigh channel models. From Fig. 15 it has been observed that LS CE gives better performance only for the static AWGN channel as FER decreases suggestively for the AWGN channel that represents in blue dotted line. On the other hand, Doppler frequency changing environment there are no changes in FER enhancement more over it affects the receiver performance severely for high spread Doppler shift. The channel response derived from above equations are used for the equalization by considering that the channel is stationary for the whole packet. If the transmitter or receiver is in motion then there will be the presence of frequency and phase offset of the transmitting signal. As a result, at the receiver end, LS estimation is not efficient to minimize the FER. 


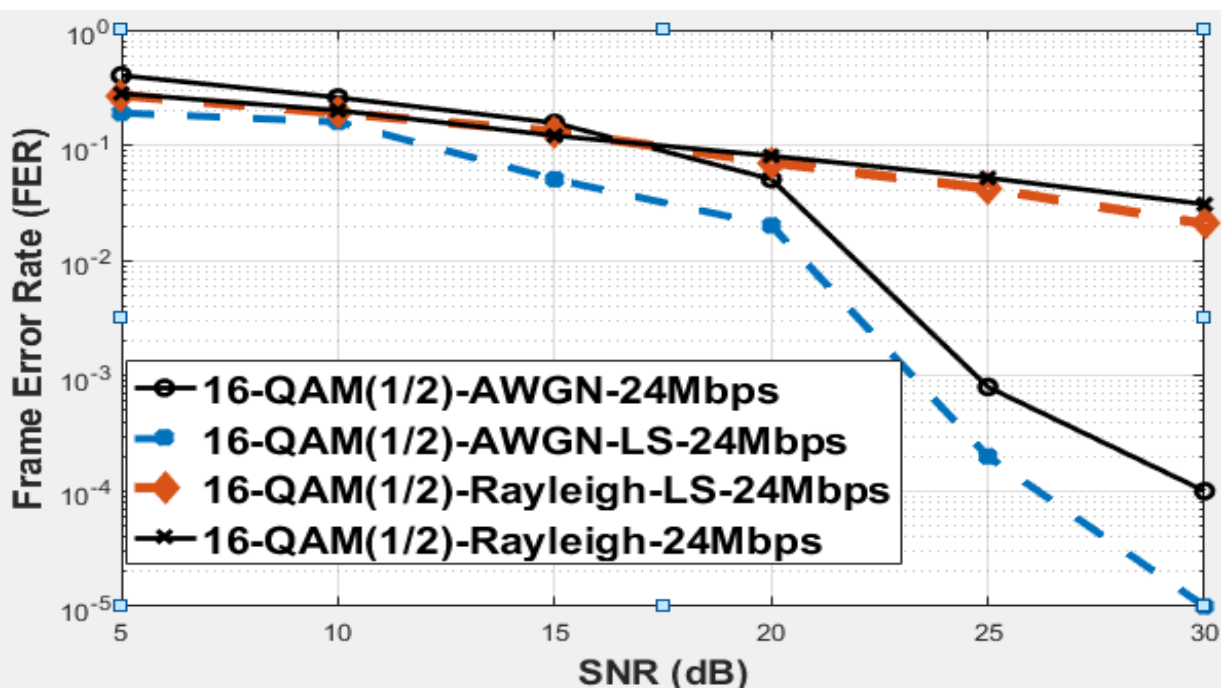

Fig. 15. FER performance of LS CE for 16-QAM over AWGN and Rayleigh fading channel

$$
\left(f_{d} T_{s}=0.0133\right)
$$

To improve the estimator performances iterative based CE method has been investigated known as Least Mean Square (LMS) algorithm. The rapidly changing time varying channel requires adaptive estimation process as it has to continuously up-to-date its filter coefficients following the channel conditions. Adaptive algorithm enhances the performance by minimizing the mean square error (MSE) between the desired equalizer output and the actual equalizer output [8]. The unknown multipath fading channel has to be equalized and properly estimated by using an adaptive filtering algorithm which has the knowledge of prior transmission of data known as a periodic training sequence. This adaptive process is continued until the MSE of LMS algorithm is zero and equalizer are converged. Let us consider that signal $x(n)$ is transmitted over the time varying wireless fading channel which is described through an L-tapped FIR filter having time varying complex coefficients $a_{n L}$. The received signal at the channel filter output can be expressed as:

$$
y(n)=\sum_{l=0}^{L-1} a(n, l) x(n-l)+w(n)
$$

Here, $x(n-L)$ is the complex symbol strained from the $x$ constellation of the $\mathrm{L}$ paths at time instant $n-L, a(n, l)$ is the complex channel tapped coefficients and $w(n)$ is the AWGN noise. The LMS algorithm can be mathematically expressed as the vector notation as given below:

The transmitted signal, $x(n)=[x(n) x(n-1) x(n-2) \ldots \ldots \ldots \ldots x(n-L)]^{T}$, the received signal, $y(n)=[y(n) \quad y(n-1) \quad y(n-2) \ldots \ldots \ldots \ldots y(n-L)]^{T}$, the filter complex coefficients at different tap positions, $a_{n}=$

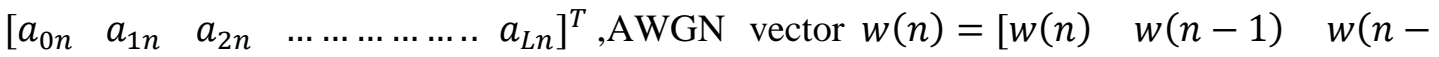
2) $\ldots . w(n-L)]^{T}$, the adaptive filter tap weights is $\widehat{a_{n}}$. The received signal can be written in vector form as $y(n)=a_{n}^{T} x(n)$. The error signal that has to be minimized adaptively and update the filter tap weights accordingly in the estimation process can be expressed as 
$e(n)=d(n)-y(n)=d(n)-a_{n}{ }^{T} x(n)$, here $d(n)$ is the predefined known transmitted training symbol that basically used for channel equalization and error approximation. The error signal $e(n)$ requires to compute the cost function $E\left[e(n) e^{*}(n)\right]$ or MSE function and minimization of the cost function is done by applying the steepest descent algorithm. By detecting the prior known training sequence adaptive filter can compute the possible error signal and minimize the cost function by driving the tap weights through iteration process until the next training sequence is sent. At time instant $n$ the mean square error (MSE) can be calculated as given below:

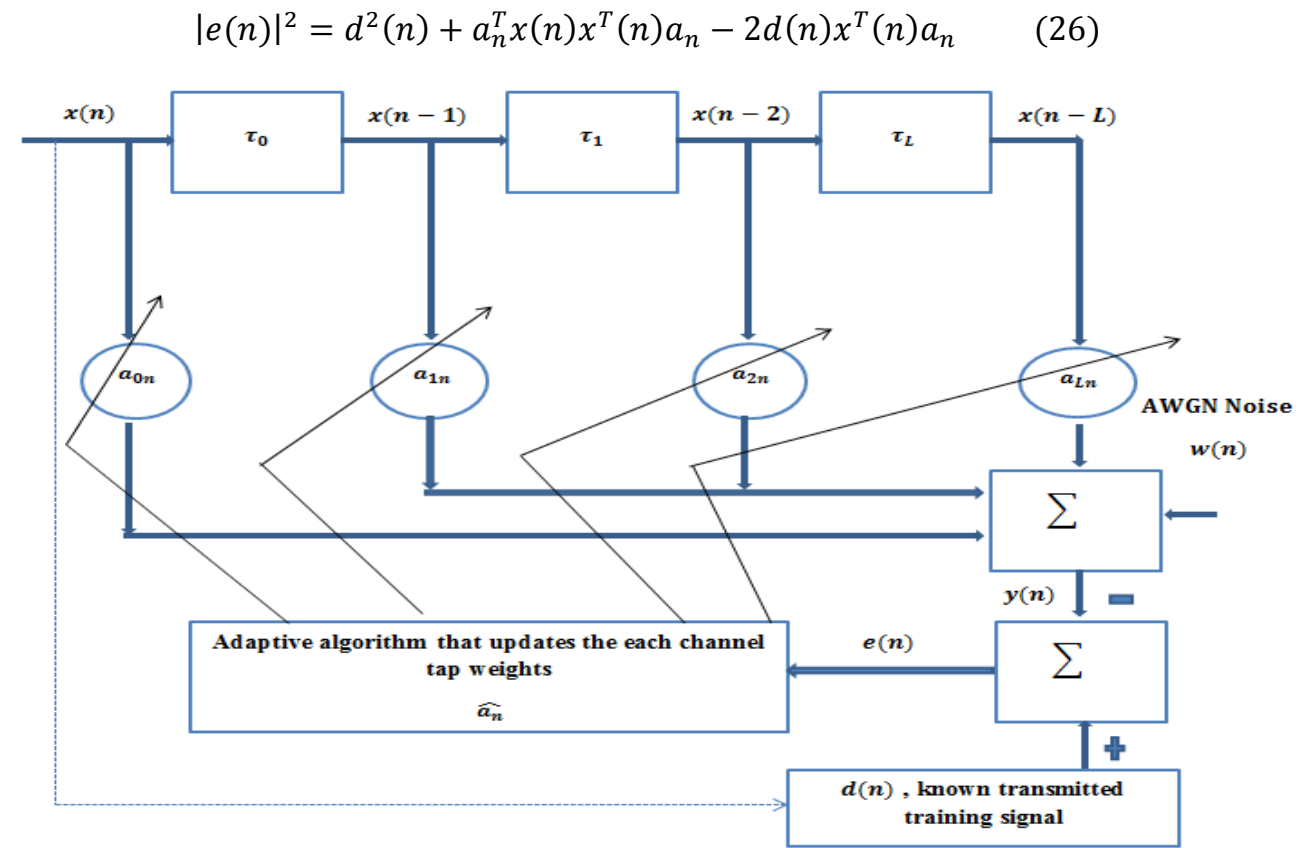

Fig. 16. Adaptive CE using LMS algorithm

Now taking the expected mean of the above equation MSE can be written as [25]:

$$
\begin{gathered}
E\left[|e(n)|^{2}\right]=E\left[d^{2}(n)\right]+a_{n}^{T} E\left[x(n) x^{T}(n)\right] a_{n} \\
-2 E\left[d(n) x^{T}(n)\right] a_{n}
\end{gathered}
$$

The cross correlation vector $P_{C C}$ between the desired response $d(n)$ and the input signal $x(n)$ can be expressed as:

$$
\begin{aligned}
P_{C C}=E[d(n) x(n)] & \\
& =E[d(n) x(n) \\
& -L)]^{T}
\end{aligned}
$$

The input correlation matrix $R_{C R}$ is defined as given below: 


$$
\begin{aligned}
& R_{C R}=E\left[x(n) x^{T}(n)\right] \\
& =\left[\begin{array}{cccc}
x^{2}(n) & x(n) x(n-1) & x(n) x(n-2) \ldots \ldots \ldots \ldots & x(n) x(n-L) \\
x(n-1) x(n) & x^{2}(n-1) & x(n-1) x(n-2) \ldots \ldots \ldots & x(n-1) x(n-L) \\
x(n-L) x(n) & x(n-L) x(n-1) & x(n-L) x(n-2) \ldots \ldots \ldots & x^{2}(n-L)
\end{array}\right]
\end{aligned}
$$

The MSE can be written as $M S E=E\left[x^{2}(n)\right]+a_{n}^{T} R_{C R} a_{n}-2 P_{C C}^{T} a_{n}$. Minimization of this MSE equation in terms of tap weight $a_{n}$ is conceivable to adaptively tune the equalizer to curtail the ISI in the received signal. Let the cost function $J\left(a_{n}\right)$ as the MSE which is basically the function of tap gain vector $a_{n}$. Minimization process is done by taking the derivative and putting the derivative equal to zero as given below:

$$
\begin{aligned}
\frac{\partial}{\partial a_{n}} J\left(a_{n}\right)=-2 P_{C C}^{n}+2 R_{C R}^{n n} a_{n}=0 \\
P_{C C}^{n}=R_{C R}^{n n} \widehat{a_{n}}, \quad \hat{a}_{n}=R_{C R}^{n n-1} P_{C C}^{n}
\end{aligned}
$$

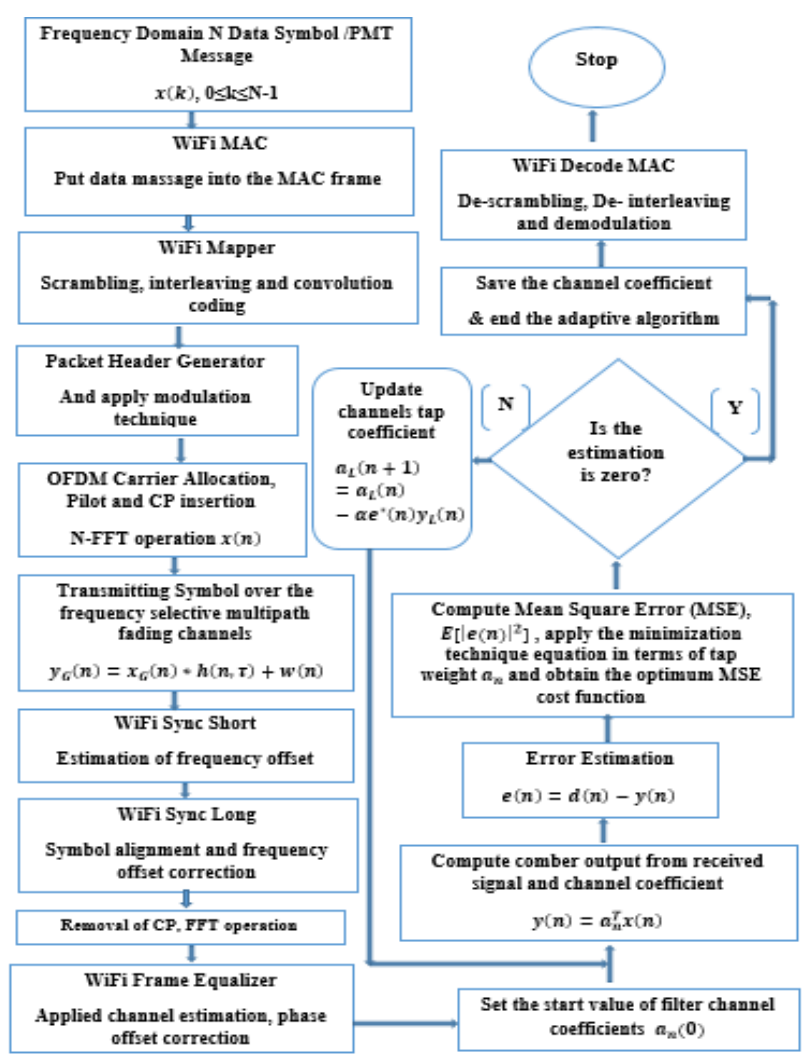

(a) Implementation of IEEE 802.11 transceiver using LMS CE

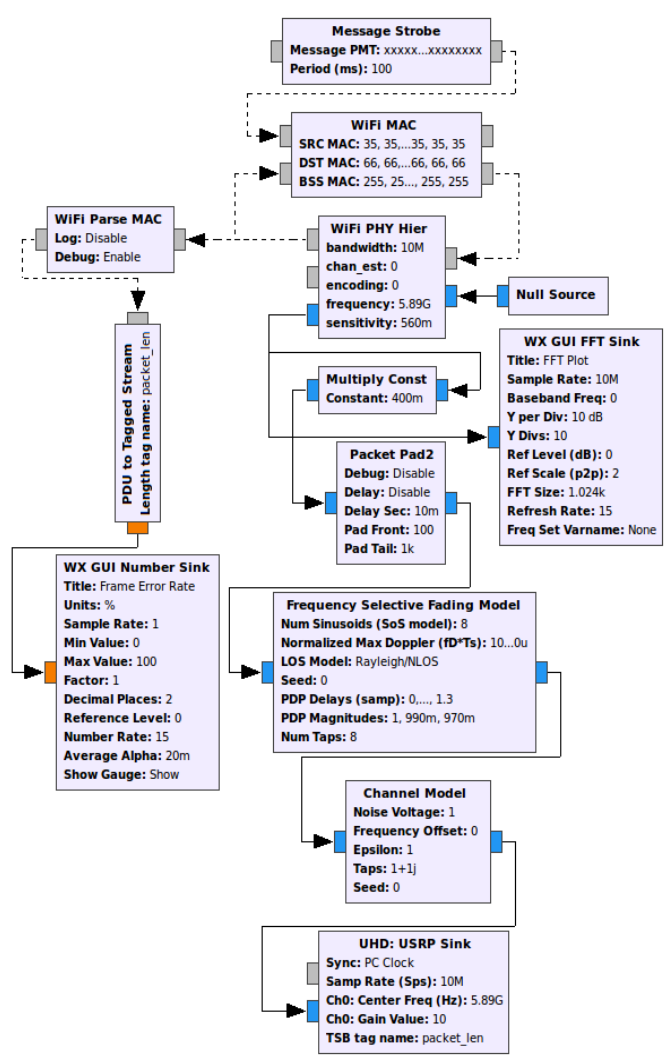

(b) IEEE 802.11p Transmitter in GRC 


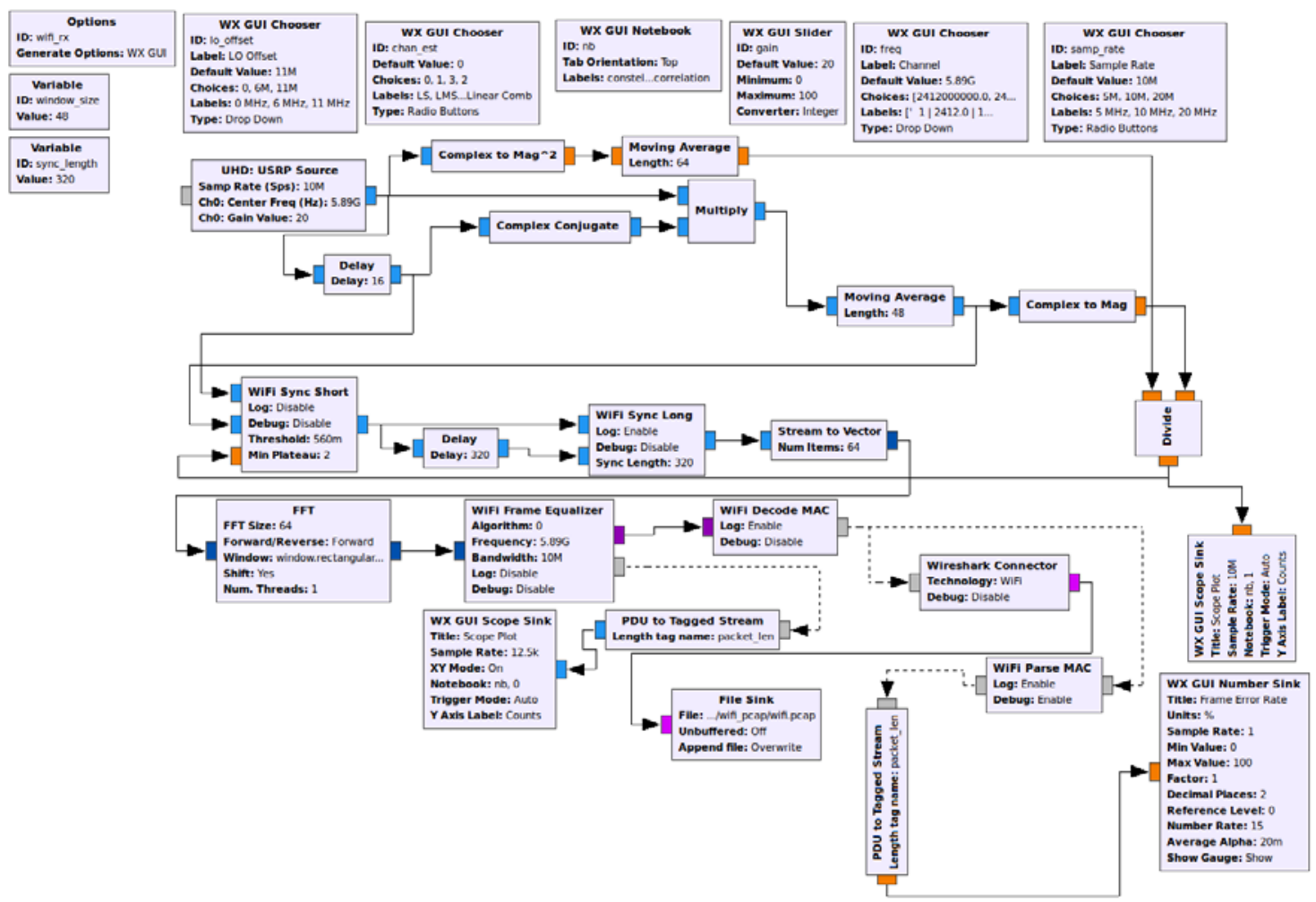

(c) IEEE $802.11 p$ Receiver in GRC

Fig. 17. IEEE 802.11p GNU Radio Implementation Blocks

Eventually, the Minimum Mean Square Error (MMSE) or the optimal cost function in terms of optimal tap gain vector $\hat{a}_{n}$ can be written as given below:

$$
J_{o p t}=J\left(\hat{a}_{n}\right)=E\left[d(n) d^{*}(n)\right]-P_{C C}^{n}{ }^{T} \hat{a}_{n}
$$

The LMS is computed through the $n$ iterative processes of minimization of the cost function to get the convergence state of the equalizer and adaptive adjustment of tap weight vector which can be summarized by the equations given below:

$$
\begin{aligned}
& y(n)=a^{T}(n) x(n) \\
& e(n)=x(n)-y(n) \\
& a_{L}(n+1)=a_{L}(n)-\alpha e^{*}(n) y_{L}(n)
\end{aligned}
$$

$\mathrm{L}$ is the number of delay taps in the fading channel model, $\alpha$ is the step size which defines the rate of convergence and stability of the LMS algorithm, $a_{L}(n+1)$ denotes the weight vector to be computed at iteration $(n+1)$. The stability of $\alpha$ is defined within the range $0<\alpha<\frac{2}{\sum_{j=1}^{L} \lambda_{j}}$, here $\lambda_{j}$ is the $j$ th Eigen value of the convergence matrix $R_{C R}^{n n}$. 
Fig. 18 (a) illustrates the FER performance after applying LMS CE technique during data symbol transmission over Rayleigh (NLOS) and Rician (LOS) distributed fading channels. It has been noticed that the adaptive LMS CE technique reduced FER at the receiver that is represented in dotted line in Fig. 19 (a) for both the channel models with compared to the not equalized FER which are represented in solid line in Fig. 19 (a). Here the receiver has given significantly less FER when the SNR approaches at $25 \mathrm{~dB}$.

Fig. 18 (b) illustrates the LMS CE estimator performance for different normalized Doppler frequency values in Rician (LOS) fading channel. It has been observed that with increasing the relative speed $v \in\{84,120,150\}$ meter $(m) /$ second $(s)$ and corresponding normalized Doppler frequency $f_{d} T_{s} \in\{0.0133,0.0188,0.0235\}$, the FER has significantly lower values while SNR approaches at $25 \mathrm{~dB}$.

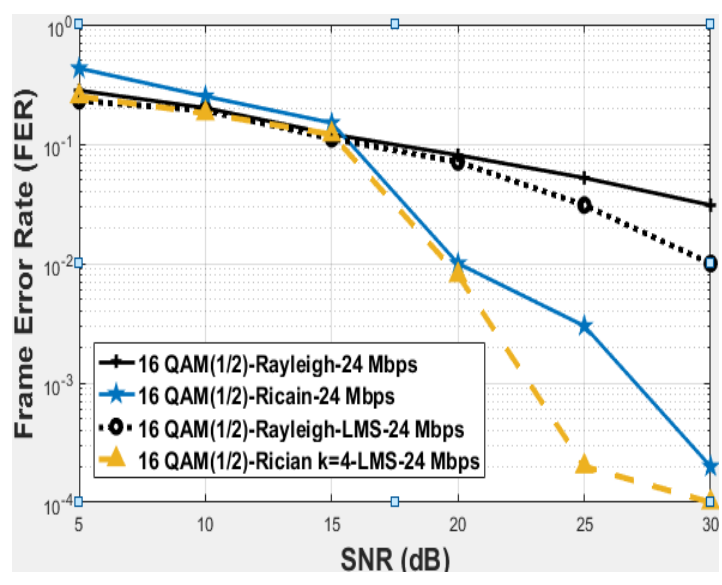

(a) FER vs. SNR, 16-QAM, $f_{d} T_{s}=0.0133$

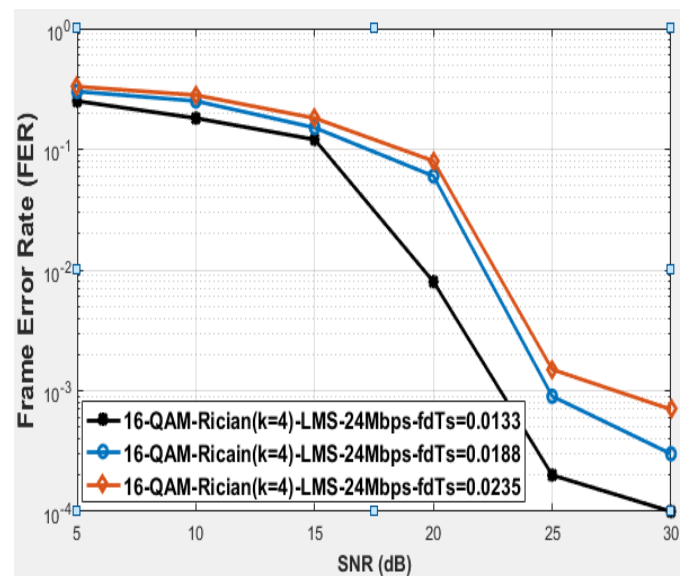

(b) FER vs. SNR, 16-QAM, $f_{d} T_{s} \in\{0.0133,0.0188,0.0235\}$

Fig. 18. LMS CE estimator performance comparisons for 16-QAM, 24 Mbps data rate, Rayleigh and Rician channel model; (a) FER vs. SNR for $f_{d} T_{s}=0.0133$; (b) FER vs. SNR with different normalized Doppler shift $f_{d} T_{s}=0.0133,0.0188$ and 0.0235 respectively

\section{Conclusion}

At the receiver, LS and training symbol based adaptive LMS CE techniques have been applied in GNU Radio and USRP N200 platform to minimize the FER of IEEE $802.11 \mathrm{a} / \mathrm{g} / \mathrm{p}$ standard transceiver while data pass through the wireless fading channel models developed in GRC. More precise approximation of SNR is done by modifying the SNR calculation process of YANS and NIST error rate model as mentioned in the equation no. (15) to get accurate BER and FER of IEEE $802.11 \mathrm{a} / \mathrm{g} / \mathrm{p}$ standard transceiver. The wireless fading channels can be expressed as the FIR filter with delay taps and complex tap coefficients that actually gives impulse response by adding certain distortion to the amplitude and phase of the transmitted symbol. Here it has been observed that for fading channels LMS CE techniques give better performance as it adjusts with the channel condition through the training based adaptive minimization process of error cost function. According to the FER vs. SNR curve analysis, for the both of fading channel models known as Rayleigh and Rician, assigned Power Delay Profile (PDP), normalized Doppler shift 0.0133, chosen modulation 
scheme 16 QAM and data rate 24 Mbps, the receiver has given suggestively less FER while SNR approaches at $25 \mathrm{~dB}$. Therefore, according to the analysis, it has been stated that applied channel estimation and equalization technique enhances the receiver sensitivity.

\section{References}

[1] Bastian Bloessl, Michele Segata, Christoph Sommer and Falko Dressler, "Towards an Open Source IEEE 802.11p Stack: A Full SDR-based Transceiver in GNU Radio," in Proc. of 5th IEEE Vehicular Networking Conference (VNC 2013), Boston, MA, pp. 143-149, Dec. 2013. Article (CrossRef Link)

[2] Bastian Bloessl, Michele Segata, Christoph Sommer and Falko Dressler, "Decoding IEEE 802.11a/g/p OFDM in Software using GNU Radio," in Proc. of 19th ACM International Conference on Mobile Computing and Networking (MobiCom), Miami, FL, pp. 159-161, Oct. 2013. Article (CrossRef Link)

[3] Yan Yang, Dan Fei and Shuping Dang, "Inter-Vehicle Cooperation Channel Estimation for IEEE 802.11p V2I Communications," Journal of Communications and Networks, Vol.19, No.3, June 2017. Article (CrossRef Link)

[4] Lihua Yang, Longxiang Yang and Yan Liang, "Iterative Channel Estimation for MIMOOFDM system in Fast Time Varying Channels," KSII Transactions of Internet and Information Systems, Vol. 10, No. 9, Sept. 2016. Article (CrossRef Link)

[5] Razvan Andrei Stoica, Stefano Severi and Giuseppe Thadeu Freitas de Abreu, "On Prototyping IEEE 802.11p Channel Estimators in Real World Environments Using GNU Radio," IEEE Intelligent Vehicles Symposium (IV), Gothenburg, Sweden, June, 2016.

Article (CrossRef Link)

[6] Aida Zaier and Ridha Bouallegue, "A Full Performance Analysis of Channel Estimation Methods for Time Varying OFDM Systems," International Journal of Mobile Network Communication \& Telematics, Vol.1, No.2, Dec. 2011. Article (CrossRef Link)

[7] Jusnaini Muslimin, A.L. Asnawi, A.F. Ismail, H.A. Mohd Ramli, A.Z. Jusoh, S. oorjannah and Nor F. M. Azmin, "Basic Study of OFDM with Multipath Propagation Model in GNU platform," IEEE Conference on Wireless Sensors, 2015. Article (CrossRef Link)

[8] ElSayed A. Ahmed and Mohamed M. Khairy, "Semi-adaptive Channel Estimation Technique for LTE Systems," IEEE Symposium on Computers and Communications (ISCC), 2011.

Article (CrossRef Link)

[9] Meng-Han Hsieh and Che-Ho Wei, "Channel Estimation for OFDM Systems Based on Comptype Pilot Arrangement in Frequency Selective Fading Channels," IEEE Transactions on Consumer Electronics, Vol.44, No.1, February 1998. Article (CrossRef Link)

[10] P. Fuxjager, A. Cotantini, D. Valerio, P. Castiglione, G. Zacheo, T. Zemen and F. Ricciato, "IEEE 802.11p Transmission Using GNU Radio," in Proc. of $6^{\text {th }}$ Karlsruhe Workshop on Software Radios, Karlsruhe, Germany, March 2010.

http://thomaszemen.org/papers/Fuxjaeger10-WSR-paper.pdf

[11] Ha Cheol Lee, "FER Performance in the IEEE $802.11 \mathrm{a} / \mathrm{g} / \mathrm{n}$ Wireless LAN over Fading Channel," Scientific Research on Communications and Network, 5, 10-15, 2013.

Article (CrossRef Link)

[12] M.Eng. Christopher, B.Eng. Arthur Witt and Prof. Dr. Roland Muenzner, “A new ns-3 WLAN error rate model- Definition, validation of the ns-3 implementation and comparison to physical layer measurements with AWGN channel," in Proc. of Workshop on ns-3 (WNS3), Barcelona, Spain, 2015.

https://www.nsnam.org/wp-content/uploads/2015/04/WNS3_2015_submission_34.pdf

[13] Md. Masud Rana, Jisang Kim and Won-Kyung Cho, "An Adaptive LMS Channel Estimation Method for LTE SC-FDMA Systems," International Journal of Engineering \& Technology, Vol. 10, No. 5, December 2010. http://ijens.org/101205-6464\%20IJET-IJENS.pdf 
[14] Olivier Goubet, Gwilherm Baudic, Frederic Gabry and Tobias J. Oechtering, "Low Complexity Scalable Iterative Algorithms for IEEE 802.11p Receivers,” IEEE Transactions on Vehicular Technology, 2014. Article (CrossRef Link)

[15] Md. Masud Rana and Md. Kamal Hosain, “Adaptive Channel Estimation Techniques for MIMO OFDM Systems, International Journal of Advanced Computer Science and Applications,” Vol.1, No.6, December 2010.

https://pdfs.semanticscholar.org/e120/db38120d5e756cfdde419a36be46bf4b68e3.pdf

[16] Zijun Zhao, Xiang Cheng and Miaowen, Bingli Jiao, Cheng-Xiang Wang, "Channel Estimation Schemes for IEEE 802.11p Standard," IEEE intelligent transportation systems magazine, 2013. Article (CrossRef Link)

[17] Guangyu Pei and Thomas R. Henderson, "Validation of OFDM NIST and YANS error rate model in ns-3, Boeing Research \& Technology," technical report from https://www.nsnam.org/ pei/80211ofdm.pdf

[18] Bahattin Karakaya, Huseyin Arslan, Hakan and Ali Cirpan, "Channel Estimation for LTE Uplink in High Doppler Spread," IEEE Wireless Communications and Networking Conference, 2008. Article (CrossRef Link)

[19] GNU Radio Manual and C++ API Reference (3.7.10.1) [Online]. Available: Article (CrossRef Link)

[20] Sinem Coleri, Mustafa Ergen, Anuj Puri and Ahmad Bahai, "A study of channel estimation in OFDM systems," in Proc. of IEEE 56th Vehicular Technology Conference, 2002. Article (CrossRef Link)

[21] Bastian Bloessl, IEEE 802.11 a/g/p Transceiver [Online]. Available: https://github.com/bastibl/gr-ieee802-11.git

[22] Samaneh Shooshtary, "Development of a MATLAB Simulation Environment for Vehicle-toVehicle and Infrastructure Communication Based on IEEE 802.11p,” M.S. thesis, Department of Technology and Build Environment, University of Gavle, Dec., 2008. http://www.divaportal.org/smash/get/diva2:133311/FULLTEXT01.pdf

[23] Rao, K. Deergha, "Channel Coding Technique for Wireless Communication,” Performance of digital communication over fading channels, Chapter 2, Springer, 2015.

http://www.springer.com/gp/book/9788132222910

[24] Theodore S.Rappaport, "Wireless Communication Principles and Practice," $2^{\text {nd }}$ Edition, Pearson Education, ch. 5-7, pp. 177-415, 2002.

[25] GNU Radio, the free and open source radio ecosystem, [Online]. Available: http://gnuradio.org/redmine 

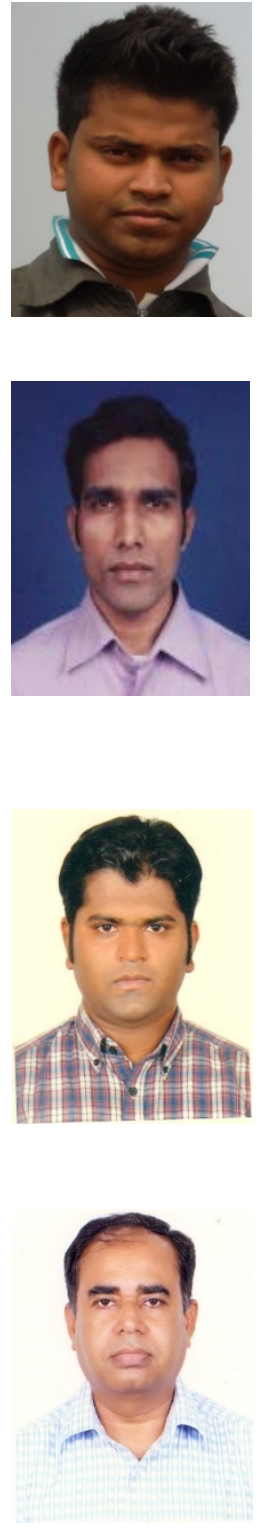

Muhammad Morshed Alam received the B.Sc. degree in Electrical and Electronic Engineering (EEE) from Independent University, Bangladesh (IUB) in 2013. At present, he is doing the M.Sc. degree in Electrical and Electronic Engineering (EEE) at Islamic University of Technology (IUT), Dhaka. His research interest includes cognitive radio networks, wireless sensor and ad-hoc networks, VoIP QoS, integration and security, Linux and UNIX and communication protocols: SIP, H.323, SIGTRAN. He is working as Senior Network Engineer at Amber IT which is a leading internet service provider of Bangladesh. He has been published 5 research manuscripts in different peer-reviewed international journals and conferences.

Mohammad Rakibul Islam received the B.Sc.Engg. and M.Sc.Engg. degree in Electrical and Electronic Engineering from Bangladesh University of Engineering and Technology (BUET), Bangladesh in 1998 and 2004 respectively. He also received MBA degree in Marketing from the Institute of Business Administration (IBA) under the University of Dhaka in 2006. He received his Ph.D. degree in the department of Electronics and Radio Engineering from Kyung Hee University, South Korea in the year 2010. He joined the Department of Electrical and Electronic Engineering, Islamic University of Technology (IUT) as a faculty on 1999 and serving as a Professor there. His research interests include the cooperative technique for wireless sensor networks, LDPC and QC-LDPC codes, secrecy capacity, Photonics and other wireless applications.

Muhammad Yeasir Arafat is a Ph.D. student in Computer Engineering at Chosun University, South Korea. He got Korean Government Scholarship (KGSP) at 2016. He received M.Sc. degree in Computer Network and Communications engineering from Independent University, Bangladesh, in 2014 and B.Sc. degree in Electronic and Telecommunications engineering from Independent University, Bangladesh, in 2012. His research interests fall into the areas of wireless networking, UAV networks, sensor networks and the design of routing protocols for vehicular and flying ad hoc networks. He has been published over 6 papers in different peer-reviewed international journals and 5 papers on national and international conferences. Mr. Yeasir is a member of the Institute of Electrical and Electronic Engineers (IEEE).

Feroz Ahmed received the B.Sc. degree in electrical and electronic engineering from Rajshahi University of Engineering and Technology, Bangladesh, in 1995, the M.Sc. degree in electrical engineering from Universiti Teknologi Malaysia, Malaysia, in 1998, and the Ph.D. degree in communication engineering from the University of ElectroCommunication, Tokyo, Japan in 2002. From 2002 to 2005, he worked as a postdoctoral research fellow at Gunma University, Gunma, and the University of Electro Communication, Tokyo, Japan. In 2005, he was appointed as Assistant Professor in the school of engineering and computer science at the Independent University, Bangladesh and became an Associate Professor in 2011. His research interests include RF and wireless communication systems, photonics and fiber-optics, software defined radio, and non-destructive testing. He has published over 60 papers in different peer review international journals and conferences. Dr. Ahmed is a member of the Institute of Electrical and Electronic Engineers (IEEE). 